\title{
A structural differentiation of quaternary copper argyrodites: Structure - property relations of high temperature ion conductors
}

\author{
Tom Nilges and Arno Pfitzner* \\ Institut für Anorganische Chemie, Universität Regensburg, Universitätsstraße 31, D-93040 Regensburg, Germany \\ Dedicated to Professor Dr. Hans-Jörg Deiseroth on the occasion of his $60^{\text {th }}$ birthday
}

Received August 2, 2004; accepted September 14, 2004

Copper argyrodites / Anharmonic refinements / Ion conductors / Powder diffraction structure analysis / $X$-ray diffraction

\begin{abstract}
The crystal structures of 12 argyrodite type copper compounds with the general formula $\mathrm{Cu}_{(12-n)} B^{n+} Q^{2-}{ }_{6-y} X^{-}{ }_{y} \quad(B=\mathrm{P}$, As, Si, Ge; $Q=\mathrm{S}, \mathrm{Se}$; $X=\mathrm{Cl}, \mathrm{Br}, \mathrm{I})$ were refined. The positions of the copper atoms were refined by using a non-harmonic approach. All polymorphic argyrodites were investigated in their cubic high temperature modification crystallizing in spacegroup $F \overline{4} 3 m$ (No. 216). A comprehensible way to describe the complex structures was developed based on a topological description of the rigid anion- and $B$-cation substructure as an arrangement of Frank-Kasper polyhedra. An analysis of the joint probability density function and of the one particle potentials for the copper atoms was performed to get a detailed insight in the copper distribution in these argyrodites. They can be divided into four types based on their different distribution of copper.

This classification corresponds to the physical properties of the argyrodites, especially to their ionic conductivities, which show a significant dependence on the composition.
\end{abstract}

\section{Introduction}

The argyrodites were declared as a structure family by Hahn, Schulze and Sechser (1965), and Kuhs, Nitsche and Scheunemann (1978a; 1979), and can be derived from the mineral $\mathrm{Ag}_{8} \mathrm{GeS}_{6}$ called Argyrodite (Goldschmidt, 1909; Palache, Berman and Fondel, 1944). The ternary members of the structure family have the general formula $A^{m+}{ }_{(12-n)}$ ${ }_{m}{ }^{n+}{ }^{2-}{ }_{6} \cdot A^{m+}$ are restricted to $\mathrm{Cu}^{+}, \mathrm{Ag}^{+}, \mathrm{Cd}^{2+}$ (Krebs and Mandt, 1972; Susa and Steinfink, 1971) and $\mathrm{Hg}^{2+}(\mathrm{Gu}-$ lay, Olekseyuk and Parasyuk, 2002) but the $B$-cation can be substituted by tetravalent $\mathrm{Ti}$ and pentavalent $\mathrm{Nb}$ or $\mathrm{Ta}$ (e.g.

* Correspondence author (e-mail: arno.pfitzner@chemie.uni-regensburg.de) in $\operatorname{Ag}_{(12-n)} B^{n+} Q_{6}, Q=\mathrm{S}$, Se) (Onoda, Wada, Hiroaki and Tansho, 1998; Wada, 1992; Wada, Sato, Onoda, Adams, Tansho and Ishii, 2002) and most of the group 13 to group 15 elements of the periodic table (trivalent $\mathrm{Al}$ and Ga (Gaudin, Deiseroth and Zaiss, 2001; Tansho, Wada, Ishii and Onoda, 1996) tetravalent $\mathrm{Si}, \mathrm{Ge}$ and $\mathrm{Sn}$ (Matje and Schön, 1980) and pentavalent P and As. Sulfur can be substituted by selenium or tellurium $(Q=\mathrm{S}, \mathrm{Se}, \mathrm{Te})$.

Intense research on the ternary argyrodites was performed in the last three decades because of their interesting physical properties, especially ion conductivity (e.g. $\mathrm{Ag}_{6.69} \mathrm{GeSe}_{5} \mathrm{I}_{0.69}$ : Belin, Aldon, Zerouale, Belin and Ribes, 2001) and photovoltaic and illumination effects (Batirov, Fridkin, Nitsche and Verkhovskaya, 1982).

The high ionic conductivity results from order-disorder phenomena in the substructure of the $A$-cations. Contrary, the substructure of the $B$-cation and the anions is rigid. However, one chalcogen atom can be substituted by a halogen atom resulting in compositions like $A^{m+}{ }_{(12-n / m)} B^{n+} Q^{2-}{ }_{6-y} X^{-}{ }_{y} \quad(X=\mathrm{Cl}, \mathrm{Br}, \mathrm{I})$. Starting from the disordered high-temperature modification, which is the aristo-type of the structure family, several partially or fully ordered hetto-types were found. The aristo-type often shows high ionic conductivity and is therefore of particular interest for electrochemical devices like batteries and sensors. $\mathrm{Cu}_{6} \mathrm{PS}_{5} \mathrm{I}$ is the best studied quaternary copper argyrodite. Besides high ionic conductivity this material exhibits ferroelastic and nonlinear optical properties (Kuhs et al., 1979; Studenyak, Stefanovich, Kranjcec, Desnica, Azhniuk, Kovacs and Panko, 1997). The determination of the electrical properties of $\mathrm{Cu}_{7} \mathrm{GeS}_{5} \mathrm{I}$ and the comparison of them with other quaternary silver and copper argyrodites like $\mathrm{Cu}_{6} \mathrm{PS}_{5} \mathrm{I}$ and $\mathrm{Ag}_{7} \mathrm{GeS}_{5} \mathrm{I}$ by Studenyak, Kranjcec, Kovac, Desnica-Frankovic, Molnar, Panko and Slivka (2002), proved an enhancement of conductivity after heterovalent substitution of $\mathrm{P}$ with $\mathrm{Ge}$ and the corresponding change in the content of monovalent cations. The crystal structures of the quaternary copper argyrodites have been scarcely studied despite their promising properties for electrochemical devices. Herein we report the determination of the crystal structures of 12 quaternary copper argyrodites and the analysis of them with respect to ionic conductivity. 
Table 1. Details of the preparation of the quaternary copper argyrodites.

\begin{tabular}{lcl}
\hline Compound & $\begin{array}{l}\text { Annealing } \\
\text { temperature in } \mathrm{K}\end{array}$ & $\begin{array}{l}\text { Annealing } \\
\text { time in d }\end{array}$ \\
\hline $\mathrm{Cu}_{6} \mathrm{PS}_{5} \mathrm{Cl}$ & 923 & 41 \\
$\mathrm{Cu}_{6} \mathrm{PS}_{5} \mathrm{Br}$ & 923 & 41 \\
$\mathrm{Cu}_{6} \mathrm{PS}_{5} \mathrm{I}$ & 1023 & 37 \\
$\mathrm{Cu}_{6} \mathrm{AsS}_{5} \mathrm{Br}$ & 853 & 62 \\
$\mathrm{Cu}_{6} \mathrm{AsS}_{5} \mathrm{I}$ & 873 & 30 \\
$\mathrm{Cu}_{7.82} \mathrm{SiS}_{5.82} \mathrm{Br}_{0.18}$ & 1193 & 19 \\
$\mathrm{Cu}_{7} \mathrm{SiS}_{5} \mathrm{I}$ & 1173 & 19 \\
$\mathrm{Cu}_{7.49} \mathrm{SiS}_{5.49} \mathrm{I}_{0.51}$ & 1193 & 19 \\
$\mathrm{Cu}_{7.44} \mathrm{SiSe}_{5.44} \mathrm{I}_{0.56}$ & 1193 & 19 \\
$\mathrm{Cu}_{7.75} \mathrm{GeS}_{5.75} \mathrm{Br}_{0.25}$ & 1153 & 23 \\
$\mathrm{Cu}_{7} \mathrm{GeS}_{5} \mathrm{I}$ & 1123 & 25 \\
$\mathrm{Cu}_{7.52} \mathrm{GeSe}_{5.52} \mathrm{I}_{0.48}$ & 960 & 25 \\
\hline
\end{tabular}

\section{Experimental}

\section{Preparation}

The argyrodites were prepared by the reaction of stochiometric mixtures of the respective elements in evacuated silica ampoules. The starting materials were heated to $1223 \mathrm{~K}$ in case of $\mathrm{Cu}_{6} \mathrm{PS}_{5} X(X=\mathrm{Cl}, \mathrm{Br}, \mathrm{I}), 1273 \mathrm{~K}$ in case of $\mathrm{Cu}_{8-x} B Q_{6-x} X_{x}(B=\mathrm{Si}, \mathrm{Ge} ; Q=\mathrm{S}$, Se; $X=\mathrm{Br}, \mathrm{I})$ and $\mathrm{Cu}_{6} \mathrm{AsS}_{5} \mathrm{X}(X=\mathrm{Br}, \mathrm{I})$, and $1373 \mathrm{~K}$ for $\mathrm{Cu}_{7} \mathrm{SiS}_{6-x} \mathrm{I}_{x}$. After grinding the argyrodites were annealed at temperatures between $923 \mathrm{~K}$ and $1193 \mathrm{~K}$ for at least 19 days.

Details concerning the composition of the materials, the annealing temperatures and the annealing times are summarized in Table 1.

\section{X-ray powder diffraction}

All materials were investigated by X-ray powder diffraction in order to determine the resulting phases and to compare the results with those reported by Kuhs et al. (1979). The compositions calculated from single crystal X-ray data were taken to prepare phase pure samples of the respective materials. The purity was checked using a Stoe STADIP powder diffractometer or a Siemens D5000 powder diffractometer, both equipped with a linear $5^{\circ} \mathrm{PSD}\left(\mathrm{Cu} K_{\alpha_{1}}\right.$ radiation, $\lambda=1.54051 \AA$, germanium monochromator). Table 2 summarizes the results from the phase analysis.

The comparison of the different compositions for the argyrodites leads to the conclusion that most of them show a broad stability range with respect to the anions (e.g. $\mathrm{Cu}_{8-x} \mathrm{SiS}_{6-x} X_{x} ; 0.51=x \leq 1$ ). In contrast to Kuhs we were able to reach full substitution $(x=1)$ in the case of $\mathrm{Cu}_{6} \mathrm{AsS}_{5} \mathrm{X}(\mathrm{X}=\mathrm{Br}, \mathrm{I}), \mathrm{Cu}_{7} \mathrm{SiS}_{5} \mathrm{I}$, and $\mathrm{Cu}_{7} \mathrm{GeS}_{5} \mathrm{I}$. Surprisingly, the lattice constants of $\mathrm{Cu}_{7-x} \mathrm{AsS}_{5-x} X_{x}(X=\mathrm{Br}$, I) are the same for the powder data in (Kuhs et al., 1979) and our single crystal measurements despite significant differences in their nominal compositions. In all other cases when the compositions are different from the values reported by Kuhs the lattice constants depict the expected trends.
Table 2. Lattice constants from powder X-ray diffraction at $298 \mathrm{~K}$. All materials crystallize face centred cubic.

\begin{tabular}{lcccc}
\hline Compound & $x^{a}$ & $x^{b}$ & $\begin{array}{l}\text { Lattice } \\
\text { constants in } \AA^{a}\end{array}$ & $\begin{array}{l}\text { Lattice } \\
\text { constants in } \AA^{b}\end{array}$ \\
\hline $\mathrm{Cu}_{7-x} \mathrm{PS}_{6-x} \mathrm{Cl}_{x}$ & 1.00 & 1.00 & $9.687(2)$ & $9.6828(7)$ \\
$\mathrm{Cu}_{7-x} \mathrm{PS}_{6-x} \mathrm{Br}_{x}$ & 1.00 & 1.00 & $9.723(2)$ & $9.7268(5)$ \\
$\mathrm{Cu}_{7-x} \mathrm{PS}_{6-x} \mathrm{I}_{x}$ & 1.00 & 1.00 & $9.787(2)$ & $9.7846(8)$ \\
$\mathrm{Cu}_{7-x} \mathrm{AsS}_{6-x} \mathrm{Br}_{x}$ & 0.36 & 1.00 & $9.843(3)$ & $9.8463(2)$ \\
$\mathrm{Cu}_{7-x} \mathrm{AsS}_{6-x} \mathrm{I}_{x}$ & 0.95 & 1.00 & $9.892(3)$ & $9.8989(8)$ \\
$\mathrm{Cu}_{8-x} \mathrm{SiS}_{6-x} \mathrm{Br}_{x}$ & 0.60 & 0.18 & $9.876(3)$ & $9.8356(9)$ \\
$\mathrm{Cu}_{8-x} \mathrm{SiS}_{6-x} \mathrm{I}_{x}$ & 0.90 & 1.00 & $9.930(2)$ & $9.9461(2)$ \\
& & 0.51 & & $9.8967(2)$ \\
$\mathrm{Cu}_{8-x} \mathrm{SiSe}_{6-x} \mathrm{I}_{x}$ & 0.83 & 0.56 & $10.346(2)$ & $10.2852(4)$ \\
$\mathrm{Cu}_{8-x} \mathrm{GeS}_{6-x} \mathrm{Br}_{x}$ & 0.70 & 0.25 & $9.953(3)$ & $9.9398(1)$ \\
$\mathrm{Cu}_{8-x} \mathrm{GeS}_{6-x} \mathrm{I}_{x}$ & 0.78 & 1.00 & $10.012(4)$ & $10.0181(2)$ \\
$\mathrm{Cu}_{8-x} \mathrm{GeSe}_{6-x} \mathrm{I}_{x}$ & 0.54 & 0.48 & $10.376(3)$ & $10.3799(1)$ \\
\hline
\end{tabular}

a: data from (Kuhs et al., 1979)

b: calculated from single crystal data

\section{Single crystal X-ray data collection and processing}

Intensity data for all argyrodites were recorded from suitable single crystals with an ENRAF NONIUS CAD4 or a STOE IPDS I. Both diffractometers operate with $\operatorname{MoK} K_{\alpha}$ radiation ( $\lambda=0.71073 \AA$, graphite monochromator). All data were corrected for Lorentz and polarisation effects. A numerical absorption correction was applied after the optimisation of the crystal shape based on symmetry equivalent reflections (IPDS I data) or on $\psi$-scans (CAD4 data) using the XRED and XSHAPE routines (XRED and XSHAPE, 1999). In accordance with the results from powder X-ray diffraction and with the data from Kuhs et al. (1979) a close structural relation between the different materials becomes obvious, i.e. they all crystallize in the cubic high temperature polymorph. Therefore, the structure of $\mathrm{Cu}_{6} \mathrm{PS}_{5} \mathrm{I}$ (Kuhs, 1978b), space group $F \overline{4} 3 m$ (No. 216) was chosen as a model for all structure refinements. According to this model copper is localised on the $24 g$ - and the $48 h$-site, the $B$-cation on the $4 b$-, the halide ion on the $4 a$ - and the chalcogen atoms on the $16 e$ - and $4 c$-site. In all cases the $A$-cation positions are not fully occupied in contrast to the anion- and the $B$-cation positions. However, the occupancy factors of the $A$-cation positions were refined without any restriction. In the case of a mixed occupancy of the $4 a$-position by $Q$ and $X$ the occupancy factors were restricted to full occupation. After the refinement of the atomic positions and the anisotropic displacement parameters and an extinction correction when necessary, the structure models where checked by difference Fourier analysis in order to localize the residual electron density left after the last refinement steps. Positions with significant residual electron density were added as copper positions to the structure model. Copper positions showing distances $d(\mathrm{Cu}-\mathrm{Cu})<0.4 \AA$ and high and anisotropic displacement parameters were refined using a non-harmonic approach based on a Gram-Charlier expansion (Kuhs, 1992; Zucker and Schulz, 1982) with non-harmonic displacement parameters up to the $4^{\text {th }}$ order. Only significant parameters were refined ( $3 \sigma$ cutoff) with the 
Table 3a. Parameters for the data collection of the quaternary copper silicon argyrodites. Space group $F \overline{4} 3 m$ (No. 216$) ; Z=4 ; T=298 \mathrm{~K}$.

\begin{tabular}{|c|c|c|c|c|}
\hline Compound $^{a}$ & $\mathrm{Cu}_{7.49} \mathrm{SiS}_{5.49} \mathrm{I}_{0.51}$ & $\mathrm{Cu}_{7} \mathrm{SiS}_{5} \mathrm{I}$ & $\mathrm{Cu}_{7.82} \mathrm{SiS}_{5.82} \mathrm{Br}_{0.18}$ & $\mathrm{Cu}_{7.44} \mathrm{SiSe}_{5.44} \mathrm{I}_{0.56}$ \\
\hline Refined composition & $\mathrm{Cu}_{6.80} \mathrm{SiS}_{5.49} \mathrm{I}_{0.51}$ & $\mathrm{Cu}_{6.85} \mathrm{SiS}_{5.03} \mathrm{I}_{0.97}$ & $\mathrm{Cu}_{7.88} \mathrm{SiS}_{5.82} \mathrm{Br}_{0.18}$ & $\mathrm{Cu}_{7.34} \mathrm{SiSe}_{5.44} \mathrm{I}_{0.56}$ \\
\hline Idealized composition & $\mathrm{Cu}_{7.49} \mathrm{SiS}_{5.49} \mathrm{I}_{0.51}$ & $\mathrm{Cu}_{7} \mathrm{SiS}_{5} \mathrm{I}$ & $\mathrm{Cu}_{7.82} \mathrm{SiS}_{5.82} \mathrm{Br}_{0.18}$ & $\mathrm{Cu}_{7.44} \mathrm{SiSe}_{5.44} \mathrm{I}_{0.56}$ \\
\hline$M_{\mathrm{X} \text {-ray }}$ in $\mathrm{g} \mathrm{mole}{ }^{-1}$ & 744.78 & 760.11 & 729.80 & 1001.48 \\
\hline$Z$ & 4 & 4 & 4 & 4 \\
\hline Crystal size in $\mathrm{mm}^{3}$ & $0.26 \times 0.20 \times 0.12$ & $0.24 \times 0.22 \times 0.18$ & $0.21 \times 0.16 \times 0.13$ & $0.22 \times 0.14 \times 0.05$ \\
\hline Crystal system & \multicolumn{4}{|c|}{ Cubic } \\
\hline$a$ in $\AA$ & $9.8967(2)$ & $9.9461(2)$ & $9.8356(9)$ & $10.2852(4)$ \\
\hline$V$ in $\AA^{3}$ & $969.33(3)$ & $983.92(3)$ & $951.5(2)$ & $1088.0(1)$ \\
\hline$\varrho_{\mathrm{X} \text {-ray }}$ in $\mathrm{g} \mathrm{cm}^{-3}$ & 5.102 & 5.130 & 5.093 & 6.112 \\
\hline Absorption coefficient $\mu_{\mathrm{MoK}_{\alpha}}$ in $\mathrm{mm}^{-1}$ & 18.970 & 19.091 & 19.218 & 34.094 \\
\hline Diffractometer; Scan & IPDS I; $\varphi$ & CAD4; $\omega$ & CAD $4 ; \omega$ & IPDS I; $\varphi$ \\
\hline$\varphi$-area in ${ }^{\circ} ; \Delta \varphi$ in $^{\circ}$ & $-2 \leq \varphi \leq 180 ; 2.0$ & - & - & $-2 \leq \varphi \leq 360 ; 2.0$ \\
\hline Absorption correction & \multicolumn{4}{|c|}{ Numerical; Optimized crystal shape (XRED and XSHAPE, 1999) } \\
\hline Crystal description & 11 faces & 15 faces & 15 faces & 10 faces \\
\hline Scan & - & $\psi$ & $\psi$ & \\
\hline No. images & 90 & - & - & 181 \\
\hline Time/plate in min & 12 & - & - & 8 \\
\hline $2 \theta$-range in ${ }^{\circ}$ & $7.1 \leq 2 \theta \leq 65.7$ & $7.1 \leq 2 \theta \leq 69.74$ & $7.18 \leq 2 \theta \leq 69.84$ & $6.9 \leq 2 \theta \leq 66.0$ \\
\hline \multirow[t]{3}{*}{$h k l$-range } & $-13 \leq h \leq 14$ & $-16 \leq h \leq 16$ & $-15 \leq h \leq 15$ & $-15 \leq h \leq 15$ \\
\hline & $-15 \leq k \leq 15$ & $-16 \leq k \leq 0$ & $0 \leq k \leq 15$ & $-15 \leq k \leq 15$ \\
\hline & $-15 \leq l \leq 14$ & $-16 \leq l \leq 0$ & $0 \leq l \leq 15$ & $-15 \leq l \leq 15$ \\
\hline Measured reflections & 3135 & 1173 & 1141 & 7134 \\
\hline Unique reflections; $R\left(F^{2}\right)_{\text {int }}$ & $223 ; 0.0637$ & $257 ; 0.0355$ & $251 ; 0.0281$ & $245 ; 0.0719$ \\
\hline Refinement program & \multicolumn{4}{|c|}{ JANA98 (Petricek et al., 1998) } \\
\hline$R / w R\left(I_{\mathrm{obs}}>3 \sigma\left(I_{\mathrm{obs}}\right)\right)$ & $0.0275 / 0.0553$ & $0.0265 / 0.0641$ & $0.0221 / 0.0560$ & $0.0319 / 0.0675$ \\
\hline$R / w R$ (all reflections) & $0.0321 / 0.0562$ & $0.0284 / 0.0649$ & $0.0265 / 0.0577$ & $0.0356 / 0.0682$ \\
\hline GoF & 2.93 & 1.68 & 2.0 & 2.45 \\
\hline Extinction coefficient & $1.36(7)$ & $0.69(4)$ & $0.56(3)$ & $0.77(4)$ \\
\hline Max/min. residual electron dens. in e $\AA^{-3}$ & $1.31 /-0.66$ & $0.89 /-0.40$ & $0.82 /-0.63$ & $1.29 /-0.97$ \\
\hline
\end{tabular}

a: Supplementary material: Crystallographic data (excluding structure factors) have been deposited with the Fachinformationszentrum Karlsruhe as supplementary publication CSD $414363\left(\mathrm{Cu}_{7.49} \mathrm{SiS}_{5.49} \mathrm{I}_{0.51}\right), \mathrm{CSD} 414354\left(\mathrm{Cu}_{7} \mathrm{SiS}_{5} \mathrm{I}\right), \mathrm{CSD}_{414355}\left(\mathrm{Cu}_{7.82} \mathrm{SiS}_{5.82} \mathrm{Br}_{0.18}\right)$ and $\mathrm{CSD}_{414365}$ $\left(\mathrm{Cu}_{7.44} \mathrm{SiSe}_{5.44} \mathrm{I}_{0.56}\right)$. Copies of available material can be obtained, free of charge, on application to Fachinformationszentrum Karlsruhe, D-76334 Leopoldshafen, Germany, (fax: 049-7247-808-666 or e-mail: crysdata@fiz-karlsruhe.de.) The list of $F_{\mathrm{o}} / F_{\mathrm{c}}$ data is available from the author up to one year after the publication has appeared.

JANA98 program package (Petricek and Dusek, 1998). A detailed description of the non-harmonic approach is given elsewhere (Pfitzner, 1997; Nilges, Reiser, Hong, Gaudin and Pfitzner, 2002).

Copper positions with $d(\mathrm{Cu}-\mathrm{Cu})>0.4 \AA$ were refined as split positions. All crystals were checked for inversion twinning but inversion twinning was not observed in the case of the compounds under discussion. Selected crystallographic data are summarized in Table 3a to c (crystallographic data), Table $4 \mathrm{a}$ to $\mathrm{b}$ (atomic coordinates and equivalent displacement parameters), Table $5 \mathrm{a}$ to $\mathrm{c}$ (selected bond lengths and angles). The structures were visualized using the programs DIAMOND (2001) and SCIAN (Pepke, Murray, Lyons and Hwu, 1994).

For $\mathrm{Cu}_{7.44} \mathrm{SiSe}_{5.44} \mathrm{I}_{0.56}$ a slightly modified structure model was used for the description of the anion substructure. Unusual high residual electron density was observed close to the Se 2 position when the common starting model for the Type 1 argyrodites was used. Instead of a fully occupied $4 c$ position $\mathrm{Se} 2$ was refined on a $16 e$ position close to $4 c$. To retain the composition of $\mathrm{Cu}_{7.44} \mathrm{SiSe}_{5.44} \mathrm{I}_{0.56}$ the occupancy factor of $\mathrm{Se} 2$ was restricted to 0.25 .

\section{Structure description and classification}

\section{Structure description}

The structures of the argyrodites under discussion can be described using three different strategies. In all strategies the mobile $A$-cation substructure is related to the rigid $B$ cation and anion substructure. So the rigid part of the structures will be discussed first. 
Table 3b. Crystallographic data for the quaternary copper germanium argyrodites. Space group $F \overline{4} 3 m$ (No. 216 ); $Z=4 ; T=298 \mathrm{~K}$.

\begin{tabular}{|c|c|c|c|}
\hline Compound $^{a}$ & $\mathrm{Cu}_{7.75} \mathrm{GeS}_{5.75} \mathrm{Br}_{0.25}$ & $\mathrm{Cu}_{7} \mathrm{GeS}_{5} \mathrm{I}$ & $\mathrm{Cu}_{7.52} \mathrm{GeSe}_{5.52} \mathrm{I}_{0.48}$ \\
\hline Refined composition & $\mathrm{Cu}_{7.37} \mathrm{GeS}_{5.75} \mathrm{Br}_{0.25}$ & $\mathrm{Cu}_{7.00} \mathrm{GeS}_{5} \mathrm{I}_{0.99}$ & $\mathrm{Cu}_{6.78} \mathrm{GeSe}_{5.52} \mathrm{I}_{0.48}$ \\
\hline Idealized composition & $\mathrm{Cu}_{7.75} \mathrm{GeS}_{5.75} \mathrm{I}_{0.25}$ & $\mathrm{Cu}_{7} \mathrm{GeS}_{5} \mathrm{I}$ & $\mathrm{Cu}_{7.52} \mathrm{GeSe}_{5.52} \mathrm{I}_{0.48}$ \\
\hline$M_{\mathrm{X} \text {-ray }}$ in $\mathrm{g} \mathrm{mol}^{-1}$ & 769.39 & 804.62 & 1047.23 \\
\hline$Z$ & 4 & 4 & 4 \\
\hline Crystal size in $\mathrm{mm}^{3}$ & $0.26 \times 0.22 \times 0.12$ & $0.21 \times 0.16 \times 0.15$ & $0.28 \times 0.20 \times 0.14$ \\
\hline Crystal system & \multicolumn{3}{|c|}{ Cubic } \\
\hline$a$ in $\AA$ & $9.9398(1)$ & $10.0181(2)$ & $10.3799(1)$ \\
\hline$V$ in $\AA^{3}$ & $982.04(3)$ & $1005.43(5)$ & $1118.35(5)$ \\
\hline$\varrho_{\mathrm{X} \text {-ray }}$ in $\mathrm{g} \mathrm{cm}^{-3}$ & 5.202 & 5.314 & 6.218 \\
\hline Absorption coefficient $\mu_{\mathrm{MoK}_{\alpha}}$ in $\mathrm{mm}^{-1}$ & 21.622 & 21.495 & 35.885 \\
\hline Diffractometer; Scan & CAD $4 ; \omega$ & $\mathrm{CAD} 4 ; \omega$ & CAD4; $\omega$ \\
\hline Absorption correction & \multicolumn{3}{|c|}{ Numerical, optimized crystal shape (XRED and XSHAPE, 1999) } \\
\hline Crystal description & 13 faces & 14 faces & 12 faces \\
\hline Scan & $\psi$ & $\psi$ & $\psi$ \\
\hline $2 \theta$-range in ${ }^{\circ}$ & $7.1 \leq 2 \theta \leq 69.78$ & $7.1 \leq 2 \theta \leq 69.84$ & $6.8 \leq 2 \theta \leq 69.32$ \\
\hline \multirow[t]{3}{*}{$h k l$-range } & $-16 \leq h \leq 16$ & $-16 \leq h \leq 16$ & $-16 \leq h \leq 16$ \\
\hline & $0 \leq k \leq 16$ & $-16 \leq k \leq 16$ & $0 \leq k \leq 16$ \\
\hline & $0 \leq l \leq 16$ & $-16 \leq l \leq 16$ & $0 \leq l \leq 16$ \\
\hline Measured reflections & 1173 & 1874 & 1341 \\
\hline Unique reflections; $R\left(F^{2}\right)_{\text {int }}$ & $257 ; 0.1201$ & $265 ; 0.0730$ & $290 ; 0.0452$ \\
\hline Reflections with $I>3 \sigma(I)$ & 234 & 241 & 273 \\
\hline No. parameters & 34 & 33 & 32 \\
\hline Refinement program & \multicolumn{3}{|c|}{ JANA98 (Petricek et al., 1998) } \\
\hline$R / w R\left(I_{\mathrm{obs}}>3 \sigma\left(I_{\mathrm{obs}}\right)\right)$ & $0.0427 / 0.0970$ & $0.0299 / 0.0561$ & $0.0223 / 0.0582$ \\
\hline$R / w R$ (all reflections) & $0.0473 / 0.1010$ & $0.0379 / 0.0586$ & $0.0275 / 0.0619$ \\
\hline GoF & 1.46 & 2.24 & 1.19 \\
\hline Extinction coefficient & $1.0(1)$ & $0.299(7)$ & $0.21(2)$ \\
\hline Max/min. residual electron dens. in e $\AA^{-3}$ & $0.93 /-0.91$ & $1.26 /-1.20$ & $0.71 /-0.74$ \\
\hline
\end{tabular}

a: Supplementary material: Crystallographic data (excluding structure factors) have been deposited with the Fachinformationszentrum Karlsruhe as supplementary publication CSD $414356\left(\mathrm{Cu}_{7.75} \mathrm{GeS}_{5.75} \mathrm{Br}_{0.25}\right)$, CSD $414357\left(\mathrm{Cu}_{7} \mathrm{GeS}_{5} \mathrm{I}\right)$, CSD $414364\left(\mathrm{Cu}_{7.52} \mathrm{GeSe}_{5.52} \mathrm{I}_{0.48}\right)$. Copies of available material can be obtained, free of charge, on application to Fachinformationszentrum Karlsruhe, D-76334 Leopoldshafen, Germany, (fax: 049-7247-808-666 or e-mail: crysdata@ fiz-karlsruhe.de.) The list of $F_{\mathrm{o}} / F_{\mathrm{c}}$ data is available from the author up to one year after the publication has appeared.

\section{The rigid anion and the $B$-cation substructure}

One of the most frequently used strategies is the description of the structures by a closed packed arrangement of anions and cations in between the voids. Three crystallographically different positions are forming the anion substructure of the $F \overline{4} 3 m$ argyrodites. In dependence of the composition the $4 a$ position is preferably or fully occupied by the halide ion of the quaternary argyrodite. The tetrahedral voids of the resulting face centred cubic arrangement are centred alternately by chalcogen atoms on $4 c$ or $4 d$ and a chalcogen tetrahedron around the remaining voids formed by atoms on $16 e$ (Fig. 1). The occupation of the $4 c / 4 d$ position depends on the configuration of the non centrosymmetric structure. In most cases $4 c / 4 d$ is populated by chalcogen atoms but can also be partially occupied by halogen. The center of the face centred cubic cell and each center of the cell edges is occupied by the tetrahedrally coordinated $B$-cations.

The A-cations are localized on a large number of linear, trigonal, and tetrahedrally coordinated positions. This

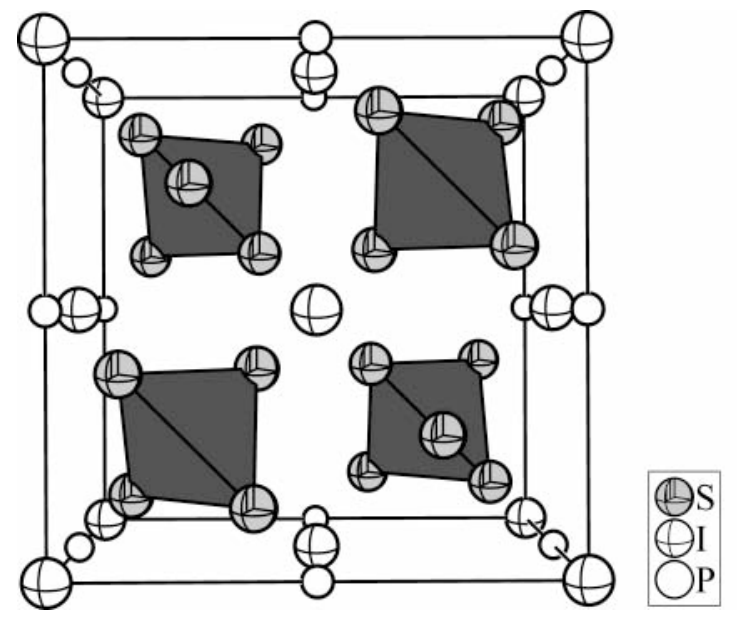

Fig. 1. B-cation and anion substructure of the cubic argyrodites illustrated for $\mathrm{Cu}_{6} \mathrm{PS}_{5} \mathrm{I}$ (space group $F \overline{4} 3 \mathrm{~m}$ ). Iodine is forming a face centred cubic arrangement. The tetrahedral voids are occupied alternately by one single chalcogen or a chalcogen tetrahedron. 
Table 3c. Data collection summary for the copper arsenic and copper phosphorus argyrodites. Space group $F \overline{4} 3 m($ No. 216$) ; Z=4 ; T=298 \mathrm{~K}$.

\begin{tabular}{|c|c|c|c|c|c|}
\hline Compound $^{a}$ & $\mathrm{Cu}_{6} \mathrm{AsS}_{5} \mathrm{I}$ & $\mathrm{Cu}_{6} \mathrm{AsS}_{5} \mathrm{Br}$ & $\mathrm{Cu}_{6} \mathrm{PS}_{5} \mathrm{I}$ & $\mathrm{Cu}_{6} \mathrm{PS}_{5} \mathrm{Br}$ & $\mathrm{Cu}_{6} \mathrm{PS}_{5} \mathrm{Cl}$ \\
\hline Refined composition & $\mathrm{Cu}_{6.06} \mathrm{AsS}_{5} \mathrm{I}$ & $\mathrm{Cu}_{5.95} \mathrm{AsS}_{5} \mathrm{Br}$ & $\mathrm{Cu}_{5.99} \mathrm{PS}_{5} \mathrm{I}$ & $\mathrm{Cu}_{6.04} \mathrm{PS}_{5} \mathrm{Br}$ & $\mathrm{Cu}_{6.16} \mathrm{PS}_{5} \mathrm{Cl}$ \\
\hline Idealized composition & $\mathrm{Cu}_{6} \mathrm{AsS}_{5} \mathrm{I}$ & $\mathrm{Cu}_{6} \mathrm{AsS}_{5} \mathrm{Br}$ & $\mathrm{Cu}_{6} \mathrm{PS}_{5} \mathrm{I}$ & $\mathrm{Cu}_{6} \mathrm{PS}_{5} \mathrm{Br}$ & \\
\hline$M_{\mathrm{X}-\text { ray }}$ in $\mathrm{g} \mathrm{mol}^{-1}$ & 743.40 & 696.40 & 699.45 & 652.45 & 608.00 \\
\hline$Z$ & \multicolumn{5}{|c|}{4} \\
\hline Crystal size in $\mathrm{mm}$ & $0.22 \times 0.20 \times 0.16$ & $0.40 \times 0.28 \times 0.14$ & $0.26 \times 0.20 \times 0.19$ & $0.24 \times 0.20 \times 0.15$ & $0.30 \times 0.23 \times 0.19$ \\
\hline$a$ in $\AA$ & $9.8989(8)$ & $9.8463(2)$ & $9.7846(8)$ & $9.7268(5)$ & $9.6828(7)$ \\
\hline$V$ in $\AA^{3}$ & $970.0(1)$ & $954.60(4)$ & $936.8(1)$ & $920.27(8)$ & $907.8(1)$ \\
\hline$\varrho_{X \text {-ray }}$ in $\mathrm{g} \mathrm{cm}^{-3}$ & 5.089 & 4.844 & 4.958 & 4.708 & 4.447 \\
\hline Absorption coefficient $\mu_{\mathrm{Mo} K_{\alpha}}$ in $\mathrm{mm}^{-1}$ & 20.491 & 21.773 & 17.883 & 19.190 & 15.346 \\
\hline Diffractometer; Scan & CAD4; $\omega$ & IPDS I; $\varphi$ & CAD4; $\omega$ & CAD $4 ; \omega$ & CAD $4 ; \omega$ \\
\hline IP distance in $\mathrm{mm}$ & - & 45 & - & - & - \\
\hline Absorption correction & \multicolumn{5}{|c|}{ Numerical. Optimized crystal shape (XRED and XSHAPE, 1999) } \\
\hline Crystal description & 16 faces & 14 faces & 17 faces & 18 faces & 18 faces \\
\hline Scan & $\psi$ & & $\psi$ & $\psi$ & $\psi$ \\
\hline No. of images & - & 181 & - & - & - \\
\hline Time/image in min & - & 5 & - & - & - \\
\hline $2 \theta$-range in ${ }^{\circ}$ & $7.1 \leq 2 \theta \leq 69.5$ & $5.1 \leq 2 \theta \leq 63.4$ & $7.2 \leq 2 \theta \leq 69.9$ & $7.2 \leq 2 \theta \leq 69.6$ & $7.2 \leq 2 \theta \leq 69.8$ \\
\hline \multirow[t]{3}{*}{$h k l$-range } & $0 \leq h \leq 15$ & $-14 \leq h \leq 14$ & $-15 \leq h \leq 15$ & $-15 \leq h \leq 15$ & $-15 \leq h \leq 15$ \\
\hline & $0 \leq k \leq 15$ & $-14 \leq k \leq 14$ & $-15 \leq k \leq 15$ & $0 \leq k \leq 15$ & $0 \leq k \leq 15$ \\
\hline & $-15 \leq l \leq 15$ & $-14 \leq l \leq 14$ & $0 \leq l \leq 15$ & $0 \leq l \leq 15$ & $0 \leq l \leq 15$ \\
\hline Measured reflections & 1169 & 5665 & 2151 & 1103 & 1081 \\
\hline Unique reflections; $R\left(F^{2}\right)_{\text {int }}$ & $256 ; 0.0425$ & $197 ; 0.0639$ & $248 ; 0.0414$ & $244 ; 0.0330$ & $241 ; 0.0619$ \\
\hline Reflections with $I>3 \sigma(I)$ & 253 & 197 & 239 & 226 & 229 \\
\hline$R / w R\left(I_{\mathrm{obs}}>3 \sigma\left(I_{\mathrm{obs}}\right)\right)$ & $0.0172 / 0.0429$ & $0.0158 / 0.0297$ & $0.0126 / 0.0341$ & $0.0170 / 0.0463$ & $0.0234 / 0.0592$ \\
\hline$R / w R$ (all reflections) & $0.0175 / 0.0432$ & $0.0158 / 0.0297$ & $0.0137 / 0.0351$ & $0.0193 / 0.0481$ & $0.0267 / 0.0612$ \\
\hline GoF & 1.14 & 2.09 & 1.25 & 1.15 & 1.17 \\
\hline Extinction coefficient & $1.18(4)$ & $0.77(3)$ & $0.30(2)$ & $0.12(1)$ & $4.6(2)$ \\
\hline Max/min. residual electron dens. in e $\AA^{-3}$ & $1.31 /-0.82$ & $0.40 /-0.67$ & $0.38 /-0.35$ & $0.22 /-0.27$ & $0.50 /-0.42$ \\
\hline
\end{tabular}

a: Supplementary material: Crystallographic data (excluding structure factors) have been deposited with the Fachinformationszentrum Karlsruhe as supplementary publication CSD $414358\left(\mathrm{Cu}_{6} \mathrm{AsS}_{5} \mathrm{I}\right), \mathrm{CSD} 414359\left(\mathrm{Cu}_{6} \mathrm{AsS}_{5} \mathrm{Br}\right), \mathrm{CSD} 414360\left(\mathrm{Cu}_{6} \mathrm{PS}_{5} \mathrm{I}\right), \mathrm{CSD}_{414362}\left(\mathrm{Cu}_{6} \mathrm{PS} \mathrm{Br}_{5}\right)$, and CSD $414361\left(\mathrm{Cu}_{6} \mathrm{PS}_{5} \mathrm{Cl}\right)$. Copies of available material can be obtained, free of charge, on application to Fachinformationszentrum Karlsruhe, D-76334 Leopoldshafen, Germany, (fax: 049-7247-808-666 or e-mail: crysdata@fiz-karlsruhe.de.) The list of $F_{\mathrm{o}} / F_{\mathrm{c}}$ data is available from the author up to one year after the publication has appeared.

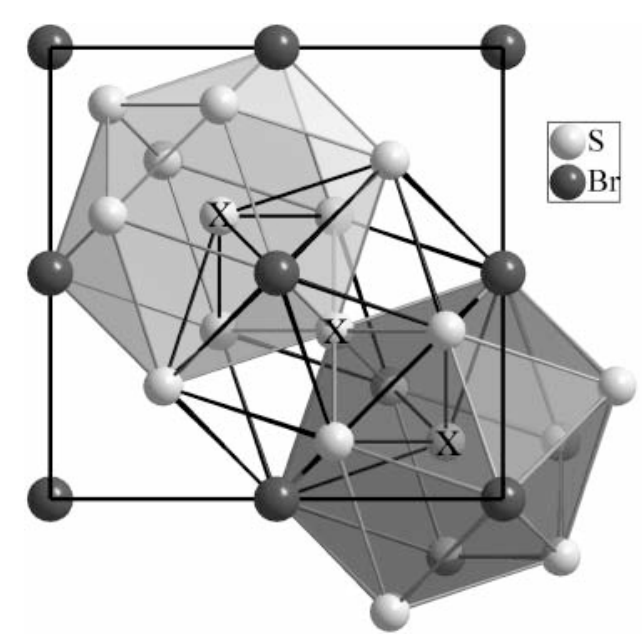

Fig. 2. Anion substructure of $\mathrm{Cu}_{6} \mathrm{PS}_{5} \mathrm{Br}$ as an example for the cubic argyrodites (space group $F \overline{4} 3 m$ ) illustrated distorted, interpenetrating icosahedra. The unit cell is drawn for clarity. approach to describe the distribution of the A-cations is rather difficult due to the high degree of disorder.

A second known strategy to describe the rigid anion substructure is based on a polyhedron representation Kuhs (1978b), described the anion substructure of the $F \overline{4} 3 m$ argyrodites by interpenetrating distorted icosahedra (see Fig. 2). The centre of the icosahedron is defined by the $16 e$ positions of the chalcogen tetrahedron. The same structural motive is known for cubic Laves phases like $\mathrm{MgCu}_{2}(\mathrm{Ohba}$, Kitano and Komura, 1984). Similar to the first strategy one ends up with the same problems describing the $A$-cation substructure.

\section{The mobile $\boldsymbol{A}$-cation substructure}

A somewhat different strategy has to be used for the description of the rigid substructure in order to achieve a more comprehensive structure description of the A-cation substructure. 
Table 4a. Fractional atomic coordinates and equivalent displacement parameters $U_{\text {eq }}$ in $\AA^{2}$ with e.s.d.s in parentheses for the Type 1 argyrodites. The maxima of the electron density of positions refined using a non-harmonic approach, the so-called mode positions, are marked with the suffix d. $U_{\text {eq }}={ }_{1 / 2}^{1} \sum \Sigma U_{i j} a^{i} a^{j} a_{i} a_{j}$

\begin{tabular}{|c|c|c|c|c|c|c|}
\hline & Pos. & sof & $x$ & $y$ & $z$ & $U_{\mathrm{eq}}$ \\
\hline \multicolumn{7}{|c|}{$\mathrm{Cu}_{6} \mathbf{P S}_{5} \mathbf{B r}$} \\
\hline $\mathrm{Cu} 1$ & $24 g$ & $0.60(2)$ & $-0.0238(1)$ & 0.25 & $y$ & $0.042(1)$ \\
\hline $\mathrm{Cu} 2$ & $48 h$ & $0.204(8)$ & $-0.0197(2)$ & $0.1910(8)$ & $y$ & $0.0332(9)$ \\
\hline S1 & $16 e$ & 1 & $0.3780(1)$ & $x$ & $x$ & $0.0125(1)$ \\
\hline $\mathrm{S} 2$ & $4 d$ & 1 & 0.75 & $x$ & $x$ & $0.0201(2)$ \\
\hline $\mathrm{Br}$ & $4 a$ & 1 & 0 & $x$ & $x$ & $0.0242(1)$ \\
\hline $\mathrm{P}$ & $4 b$ & 1 & 0 & 0.5 & $x$ & $0.0083(2)$ \\
\hline \multicolumn{7}{|c|}{$\mathrm{Cu}_{6} \mathrm{PS}_{5} \mathrm{I}$} \\
\hline $\mathrm{Cu} 1$ & $24 g$ & $0.57(7)$ & $-0.0234(2)$ & 0.25 & $y$ & $0.051(8)$ \\
\hline $\mathrm{Cu} 2$ & $48 h$ & $0.21(4)$ & $-0.0193(5)$ & $0.201(2)$ & $y$ & $0.028(1)$ \\
\hline $\mathrm{S} 1$ & $16 e$ & 1 & $0.3786(1)$ & $x$ & $x$ & $0.0105(1)$ \\
\hline $\mathrm{S} 2$ & $4 d$ & 1 & 0.75 & $x$ & $x$ & $0.0167(1)$ \\
\hline I & $4 a$ & 1 & 0 & $x$ & $x$ & $0.0154(1)$ \\
\hline$P$ & $4 b$ & 1 & 0 & 0.5 & $x$ & $0.0072(1)$ \\
\hline \multicolumn{7}{|c|}{$\mathrm{Cu}_{6} \mathrm{AsS}_{5} \mathrm{Br}$} \\
\hline $\mathrm{Cu} 1$ & $24 g$ & $0.65(1)$ & $0.0248(1)$ & 0.25 & $y$ & $0.042(1)$ \\
\hline $\mathrm{Cu} 2$ & $48 h$ & $0.171(6)$ & $0.0190(2)$ & $0.1909(7)$ & $y$ & $0.0317(8)$ \\
\hline $\mathrm{S} 1$ & $16 e$ & 1 & $0.6270(1)$ & $x$ & $x$ & $0.0109(1)$ \\
\hline S2 & $4 c$ & 1 & 0.25 & $x$ & $x$ & $0.0176(1)$ \\
\hline $\mathrm{Br}$ & $4 a$ & 1 & 0 & $x$ & $x$ & $0.0285(1)$ \\
\hline As & $4 b$ & 1 & 0 & 0.5 & $x$ & $0.0067(1)$ \\
\hline \multicolumn{7}{|c|}{$\mathrm{Cu}_{6} \mathrm{AsS}_{5} \mathrm{I}$} \\
\hline $\mathrm{Cu} 1$ & $24 g$ & $0.65(8)$ & $-0.0242(2)$ & 0.25 & $y$ & $0.056(8)$ \\
\hline $\mathrm{Cu} 2$ & $48 h$ & $0.18(4)$ & $-0.0192(9)$ & $0.200(2)$ & $y$ & $0.028(2)$ \\
\hline $\mathrm{S} 1$ & $16 e$ & 1 & 0.3733(1) & $x$ & $x$ & $0.0115(1)$ \\
\hline S2 & $4 d$ & 1 & 0.75 & $x$ & $x$ & $0.0175(2)$ \\
\hline I & $4 a$ & 1 & 0 & $x$ & $x$ & $0.0178(1)$ \\
\hline As & $4 b$ & 1 & 0 & 0.5 & $x$ & $0.0076(1)$ \\
\hline \multicolumn{7}{|c|}{$\mathrm{Cu}_{7} \mathrm{SiS}_{5} \mathbf{I}$} \\
\hline $\mathrm{Cu} 1$ & $24 g$ & $0.75(2)$ & $0.0233(1)$ & 0.25 & $y$ & $0.050(1)$ \\
\hline $\mathrm{Cu} 2$ & $48 h$ & $0.20(1)$ & $0.001(1)$ & $0.180(1)$ & $y$ & $0.085(3)$ \\
\hline $\mathrm{S} 1$ & $16 e$ & 1 & $0.6230(1)$ & $x$ & $x$ & $0.0123(2)$ \\
\hline $\mathrm{I} 2$ & $4 c$ & $0.047(5)$ & 0.25 & $x$ & $x$ & $0.0188(3)$ \\
\hline $\mathrm{S} 2$ & $4 c$ & 0.953 & 0.25 & $x$ & $x$ & 0.0188 \\
\hline I3 & $4 a$ & $0.93(1)$ & 0 & $x$ & $x$ & $0.0273(2)$ \\
\hline S3 & $4 a$ & 0.07 & 0 & $x$ & $x$ & 0.0273 \\
\hline $\mathrm{Si}$ & $4 b$ & 1 & 0 & 0.5 & $x$ & $0.0076(2)$ \\
\hline $\mathrm{Cu} 2_{d}$ & $48 h$ & & 0.0109 & 0.1925 & $y$ & \\
\hline \multicolumn{7}{|c|}{$\mathrm{Cu}_{7} \mathrm{GeS}_{5} \mathbf{I}$} \\
\hline $\mathrm{Cu} 1$ & $24 g$ & $0.77(1)$ & $0.0246(1)$ & 0.25 & $y$ & $0.053(1)$ \\
\hline $\mathrm{Cu} 2$ & $48 h$ & $0.197(6)$ & $-0.011(1)$ & $0.1762(8)$ & $y$ & $0.112(3)$ \\
\hline $\mathrm{S} 1$ & $16 e$ & 1 & $0.6265(1)$ & $x$ & $x$ & $0.0152(2)$ \\
\hline $\mathrm{I} 2$ & $4 c$ & $0.055(6)$ & 0.25 & $x$ & $x$ & $0.0215(4)$ \\
\hline S2 & $4 c$ & 0.945 & 0.25 & $x$ & $x$ & 0.0215 \\
\hline I3 & $4 a$ & $0.947(9)$ & 0 & $x$ & $x$ & $0.0279(2)$ \\
\hline S3 & $4 a$ & 0.053 & 0 & $x$ & $x$ & 0.0279 \\
\hline $\mathrm{Ge}$ & $4 b$ & 1 & 0 & 0.5 & $x$ & $0.0095(1)$ \\
\hline $\mathrm{Cu} 2_{d}$ & $48 h$ & & 0.0116 & 0.1917 & $y$ & \\
\hline \multicolumn{7}{|c|}{$\mathbf{C u}_{7.44} \operatorname{SiSe}_{5.44} \mathbf{I}_{0.56}$} \\
\hline $\mathrm{Cu} 1$ & $24 g$ & $0.58(1)$ & $0.0292(2)$ & 0.25 & $y$ & $0.069(2)$ \\
\hline $\mathrm{Cu} 2$ & $48 h$ & $0.321(8)$ & $0.0106(8)$ & $0.1684(6)$ & $y$ & $0.078(2)$ \\
\hline $\mathrm{Se} 1$ & $16 e$ & 1 & $0.6262(1)$ & $x$ & $x$ & $0.0144(1)$ \\
\hline $\mathrm{Se} 2$ & $16 e$ & 0.25 & $0.244(2)$ & $0.256(2)$ & $y$ & $0.028(1)$ \\
\hline I3 & $4 a$ & $0.56(2)$ & 0 & $x$ & $x$ & $0.0298(3)$ \\
\hline $\mathrm{Se} 3$ & $4 a$ & 0.44 & 0 & $x$ & $x$ & 0.0298 \\
\hline $\mathrm{Si}$ & $4 b$ & 1 & 0 & 0.5 & $x$ & $0.0117(3)$ \\
\hline $\mathrm{Cu} 2_{d}$ & $48 h$ & & 0.0160 & 0.1815 & $y$ & \\
\hline
\end{tabular}


Table 4b. Fractional atomic coordinates and equivalent displacement paramters $U_{\mathrm{eq}}$ in $\AA^{2}$ with e.s.d.s in parentheses for the argyrodites of Type 2,3 , and 4 . The maxima of the electron density of positions refined using a non-harmonic approach, the so-called mode positions, are marked with the suffix $d . U_{i j}=1 / 2 \sum \sum U_{i j} a^{i} a^{j} a_{i} a_{j}$

\begin{tabular}{|c|c|c|c|c|c|c|}
\hline & Pos. & sof & $x$ & $y$ & $z$ & $U_{\text {eq }}$ \\
\hline \multicolumn{7}{|c|}{ Type 2} \\
\hline \multicolumn{7}{|c|}{$\mathbf{C u}_{7.49} \mathrm{SiS}_{5.49} \mathbf{I}_{0.51}$} \\
\hline $\mathrm{Cu} 1$ & $24 g$ & $0.617(7)$ & $0.0244(1)$ & 0.25 & $y$ & $0.0476(5)$ \\
\hline $\mathrm{Cu} 2$ & $48 h$ & $0.221(4)$ & $0.0045(4)$ & $0.1768(5)$ & $y$ & $0.070(1)$ \\
\hline $\mathrm{Cu} 3$ & $16 e$ & $0.112(8)$ & $0.1218(6)$ & $x$ & $x$ & $0.113(6)$ \\
\hline $\mathrm{S} 1$ & $16 e$ & 1 & $0.6233(1)$ & $x$ & $x$ & $0.0086(1)$ \\
\hline $\mathrm{S} 2$ & $4 c$ & 1 & 0.25 & $x$ & $x$ & $0.0165(2)$ \\
\hline $\mathrm{I} 3$ & $4 a$ & $0.508(1)$ & 0 & $x$ & $x$ & $0.0185(1)$ \\
\hline S3 & $4 a$ & 0.492 & 0 & $x$ & $x$ & 0.0185 \\
\hline $\mathrm{Si}$ & $4 b$ & 1 & 0 & 0.5 & $x$ & $0.0063(2)$ \\
\hline $\mathrm{Cu} 2_{d}$ & $48 h$ & & 0.0119 & 0.1911 & $y$ & \\
\hline \multicolumn{7}{|c|}{$\mathrm{Cu}_{6} \mathbf{P S}_{5} \mathrm{Cl}$} \\
\hline $\mathrm{Cu} 1$ & $24 g$ & $0.531(6)$ & $0.0246(1)$ & 0.25 & $y$ & $0.0351(5)$ \\
\hline $\mathrm{Cu} 2$ & $48 h$ & $0.229(4)$ & $0.0170(3)$ & $0.1805(3)$ & $y$ & $0.0403(7)$ \\
\hline $\mathrm{Cu} 3$ & $16 e$ & $0.067(7)$ & $0.1160(7)$ & $x$ & $x$ & $0.061(4)$ \\
\hline $\mathrm{S} 1$ & $16 e$ & 1 & $0.6223(1)$ & $x$ & $x$ & $0.0127(1)$ \\
\hline S2 & $4 c$ & 1 & 0.25 & $x$ & $x$ & $0.0208(2)$ \\
\hline $\mathrm{Cl}$ & $4 a$ & 1 & 0 & $x$ & $x$ & $0.0350(3)$ \\
\hline $\mathrm{P}$ & $4 b$ & 1 & 0 & 0.5 & $x$ & $0.0082(2)$ \\
\hline $\mathrm{Cu} 2_{d}$ & $48 h$ & & 0.0185 & 0.1847 & $y$ & \\
\hline \multicolumn{7}{|c|}{ Type 3} \\
\hline \multicolumn{7}{|c|}{$\mathrm{Cu}_{7.82} \mathrm{SiS}_{5.82} \mathrm{Br}_{0.18}$} \\
\hline $\mathrm{Cu} 1$ & $24 g$ & $0.546(5)$ & $-0.0245(1)$ & 0.25 & $y$ & $0.0442(5)$ \\
\hline $\mathrm{Cu} 2$ & $48 h$ & $0.236(7)$ & $-0.0345(5)$ & $0.1679(5)$ & $y$ & $0.0663(9)$ \\
\hline $\mathrm{Cu} 3$ & $48 h$ & $0.148(7)$ & $0.035(2)$ & $0.1543(7)$ & $y$ & $0.080(4)$ \\
\hline $\mathrm{S} 1$ & $16 e$ & 1 & $0.3763(1)$ & $x$ & $x$ & $0.0122(1)$ \\
\hline S2 & $4 d$ & 1 & 0.75 & $x$ & $x$ & $0.0192(2)$ \\
\hline $\mathrm{Br} 3$ & $4 a$ & $0.181(9)$ & 0 & $x$ & $x$ & $0.0317(3)$ \\
\hline S3 & $4 a$ & 0.819 & 0 & $x$ & $x$ & 0.0317 \\
\hline $\mathrm{Si}$ & $4 b$ & 1 & 0 & 0.5 & $x$ & $0.0080(2)$ \\
\hline $\mathrm{Cu} 2_{d}$ & $48 h$ & & -0.0206 & 0.1783 & $y$ & \\
\hline \multicolumn{7}{|c|}{$\mathrm{Cu}_{7.75} \mathrm{GeS}_{5.75} \mathrm{Br}_{0.25}$} \\
\hline $\mathrm{Cu} 1$ & $24 g$ & $0.52(2)$ & $-0.0256(3)$ & 0.25 & $y$ & $0.037(1)$ \\
\hline $\mathrm{Cu} 2$ & $48 h$ & $0.21(1)$ & $-0.036(1)$ & $0.175(2)$ & $y$ & $0.082(5)$ \\
\hline $\mathrm{Cu} 3$ & $48 h$ & $0.15(1)$ & $0.037(3)$ & $0.156(1)$ & $y$ & $0.083(7)$ \\
\hline $\mathrm{S} 1$ & $16 e$ & 1 & $0.3730(1)$ & $x$ & $x$ & $0.0145(2)$ \\
\hline S2 & $4 d$ & 1 & 0.75 & $x$ & $x$ & $0.0213(3)$ \\
\hline $\mathrm{Br} 3$ & $4 a$ & $0.25(3)$ & 0 & $x$ & $x$ & $0.0296(7)$ \\
\hline S3 & $4 a$ & $0.75(3)$ & 0 & $x$ & $x$ & $0.0296(7)$ \\
\hline $\mathrm{Ge}$ & $4 b$ & 1 & 0 & 0.5 & $x$ & $0.0091(2)$ \\
\hline $\mathrm{Cu} 2_{d}$ & $48 h$ & & -0.0224 & 0.1849 & $y$ & \\
\hline \multicolumn{7}{|c|}{ Type 4} \\
\hline \multicolumn{7}{|c|}{$\mathrm{Cu}_{7.52} \mathrm{GeSe}_{5.52} \mathbf{I}_{0.48}$} \\
\hline $\mathrm{Cu} 1$ & $24 g$ & $0.53(1)$ & $-0.0292(3)$ & 0.25 & $y$ & $0.061(2)$ \\
\hline $\mathrm{Cu} 2$ & $48 h$ & $0.270(6)$ & $-0.0087(8)$ & $0.1726(5)$ & $y$ & $0.068(1)$ \\
\hline $\mathrm{Cu} 3$ & $24 f$ & $0.059(8)$ & 0 & $0.200(2)$ & $x$ & $0.14(2)$ \\
\hline Se1 & $16 e$ & 1 & $0.3712(1)$ & $x$ & $x$ & $0.0168(1)$ \\
\hline $\mathrm{Se} 2$ & $4 d$ & 1 & 0.75 & $x$ & $x$ & $0.0333(2)$ \\
\hline $\mathrm{I} 3$ & $4 a$ & 0.48048 & 0 & $x$ & $x$ & $0.0306(2)$ \\
\hline $\mathrm{Se} 3$ & $4 a$ & 0.519528 & 0 & $x$ & $x$ & 0.0306 \\
\hline $\mathrm{Ge}$ & $4 b$ & 1 & 0 & 0.5 & $x$ & $0.0114(1)$ \\
\hline $\mathrm{Cu} 2_{d}$ & $48 h$ & & -0.0169 & 0.1825 & $y$ & \\
\hline
\end{tabular}


Table 5a. Anisotropic displacement parameters $U_{i j}$ in $\AA^{2}$ with e.s.d.s in parentheses for the Type 1 argyrodites.

\begin{tabular}{|c|c|c|c|c|c|c|}
\hline & $U_{11}$ & $U_{22}$ & $U_{33}$ & $U_{12}$ & $U_{13}$ & $U_{23}$ \\
\hline \multicolumn{7}{|c|}{$\mathrm{Cu}_{6} \mathrm{PS}_{5} \mathrm{Br}$} \\
\hline $\mathrm{Cu} 1$ & $0.0190(4)$ & $0.054(3)$ & $U_{22}$ & 0 & 0 & $0.036(3)$ \\
\hline $\mathrm{Cu} 2$ & $0.024(1)$ & $0.038(2)$ & $U_{22}$ & $0.003(1)$ & $U_{12}$ & $0.017(2)$ \\
\hline $\mathrm{S} 1$ & $0.0125(2)$ & $U_{11}$ & $U_{11}$ & $-0.002(1)$ & $U_{12}$ & $U_{12}$ \\
\hline $\mathrm{S} 2$ & $0.0201(3)$ & $U_{11}$ & $U_{11}$ & 0 & 0 & 0 \\
\hline $\mathrm{Br}$ & $0.0242(2)$ & $U_{11}$ & $U_{11}$ & 0 & 0 & 0 \\
\hline $\mathrm{P}$ & $0.0083(3)$ & $U_{11}$ & $U_{11}$ & 0 & 0 & 0 \\
\hline \multicolumn{7}{|c|}{$\mathrm{Cu}_{6} \mathrm{PS}_{5} \mathbf{I}$} \\
\hline $\mathrm{Cu} 1$ & $0.0169(4)$ & $0.07(2)$ & $U_{22}$ & 0 & 0 & $0.05(2)$ \\
\hline $\mathrm{Cu} 2$ & $0.0209(9)$ & $0.031(2)$ & $U_{22}$ & $0.003(1)$ & $U_{12}$ & $0.013(3)$ \\
\hline $\mathrm{S} 1$ & $0.0105(1)$ & $U_{11}$ & $U_{11}$ & $-0.002(1)$ & $U_{12}$ & $U_{12}$ \\
\hline $\mathrm{S} 2$ & $0.0167(2)$ & $U_{11}$ & $U_{11}$ & 0 & 0 & 0 \\
\hline I & $0.0154(1)$ & $U_{11}$ & $U_{11}$ & 0 & 0 & 0 \\
\hline $\mathrm{P}$ & $0.0072(2)$ & $U_{11}$ & $U_{11}$ & 0 & 0 & 0 \\
\hline \multicolumn{7}{|c|}{$\mathrm{Cu}_{6} \mathrm{AsS}_{5} \mathrm{Br}$} \\
\hline $\mathrm{Cu} 1$ & $0.0172(3)$ & $0.055(2)$ & $U_{22}$ & 0 & 0 & $0.038(2)$ \\
\hline $\mathrm{Cu} 2$ & $0.0243(9)$ & $0.035(1)$ & $U_{22}$ & $-0.004(1)$ & $U_{12}$ & $0.014(2)$ \\
\hline $\mathrm{S} 1$ & $0.0109(1)$ & $U_{11}$ & $U_{11}$ & $-0.002(1)$ & $U_{12}$ & $U_{12}$ \\
\hline S2 & $0.0176(2)$ & $U_{11}$ & $U_{11}$ & 0 & 0 & 0 \\
\hline $\mathrm{Br}$ & $0.0285(1)$ & $U_{11}$ & $U_{11}$ & 0 & 0 & 0 \\
\hline As & $0.0067(1)$ & $U_{11}$ & $U_{11}$ & 0 & 0 & 0 \\
\hline \multicolumn{7}{|c|}{$\mathrm{Cu}_{6} \mathrm{AsS}_{5} \mathrm{I}$} \\
\hline $\mathrm{Cu} 1$ & $0.0184(6)$ & $0.07(2)$ & $U_{22}$ & 0 & 0 & $0.06(2)$ \\
\hline $\mathrm{Cu} 2$ & $0.023(1)$ & $0.030(3)$ & $U_{22}$ & $0.003(1)$ & $U_{12}$ & $0.010(3)$ \\
\hline $\mathrm{S} 1$ & $0.0115(2)$ & $U_{11}$ & $U_{11}$ & $-0.002(1)$ & $U_{12}$ & $U_{12}$ \\
\hline S2 & $0.0175(3)$ & $U_{11}$ & $U_{11}$ & 0 & 0 & 0 \\
\hline I & $0.0178(2)$ & $U_{11}$ & $U_{11}$ & 0 & 0 & 0 \\
\hline As & $0.0076(2)$ & $U_{11}$ & $U_{11}$ & 0 & 0 & 0 \\
\hline \multicolumn{7}{|c|}{$\mathrm{Cu}_{7} \mathrm{SiS}_{5} \mathrm{I}$} \\
\hline $\mathrm{Cu} 1$ & $0.0225(5)$ & $0.064(3)$ & $U_{22}$ & 0 & 0 & $0.040(3)$ \\
\hline $\mathrm{Cu} 2$ & $0.115(6)$ & $0.070(4)$ & $U_{22}$ & $-0.005(3)$ & $U_{12}$ & $0.015(3)$ \\
\hline $\mathrm{S} 1$ & $0.0123(3)$ & $U_{11}$ & $U_{11}$ & $-0.002(1)$ & $U_{12}$ & $U_{12}$ \\
\hline $\mathrm{I} 2 / \mathrm{S} 2$ & $0.0188(5)$ & $U_{11}$ & $U_{11}$ & 0 & 0 & 0 \\
\hline $\mathrm{I} 3 / \mathrm{S} 3$ & $0.0273(3)$ & $U_{11}$ & $U_{11}$ & 0 & 0 & 0 \\
\hline $\mathrm{Si}$ & $0.0076(4)$ & $U_{11}$ & $U_{11}$ & 0 & 0 & 0 \\
\hline \multicolumn{7}{|c|}{$\mathbf{C u}_{7} \mathbf{G e S}_{5} \mathbf{I}$} \\
\hline $\mathrm{Cu} 1$ & $0.0258(6)$ & $0.067(2)$ & $U_{22}$ & 0 & 0 & $0.037(2)$ \\
\hline $\mathrm{Cu} 2$ & $0.209(7)$ & $0.063(3)$ & $U_{22}$ & $0.013(3)$ & $U_{12}$ & $0.017(3)$ \\
\hline S1 & $0.0152(3)$ & $U_{11}$ & $U_{11}$ & $-0.003(1)$ & $U_{12}$ & $U_{12}$ \\
\hline $\mathrm{I} 2 / \mathrm{S} 2$ & $0.0215(6)$ & $U_{11}$ & $U_{11}$ & 0 & 0 & 0 \\
\hline $\mathrm{I} 3 / \mathrm{S} 3$ & $0.0279(3)$ & $U_{11}$ & $U_{11}$ & 0 & 0 & 0 \\
\hline $\mathrm{Ge}$ & $0.0095(2)$ & $U_{11}$ & $U_{11}$ & 0 & 0 & 0 \\
\hline \multicolumn{7}{|c|}{$\mathrm{Cu}_{7.44} \mathrm{SiSe}_{5.44} \mathbf{I}_{0.56}$} \\
\hline $\mathrm{Cu} 1$ & $0.027(1)$ & $0.090(5)$ & $U_{22}$ & 0 & 0 & $0.063(5)$ \\
\hline $\mathrm{Cu} 2$ & $0.093(5)$ & $0.070(2)$ & $U_{22}$ & $0.002(2)$ & $U_{12}$ & $-0.002(3)$ \\
\hline $\mathrm{Se} 1$ & $0.0144(2)$ & $U_{11}$ & $U_{11}$ & $-0.003(1)$ & $U_{12}$ & $U_{12}$ \\
\hline $\mathrm{Se} 2$ & $0.028(2)$ & $U_{11}$ & $U_{11}$ & $-0.010(4)$ & $U_{12}$ & $U_{12}$ \\
\hline $\mathrm{I} 3 / \mathrm{Se} 3$ & $0.0298(5)$ & $U_{11}$ & $U_{11}$ & 0 & 0 & 0 \\
\hline $\mathrm{Si}$ & $0.0117(6)$ & $U_{11}$ & $U_{11}$ & 0 & 0 & 0 \\
\hline
\end{tabular}

Instead of a description by interpenetrating icosahedra one can also use a set of face sharing Frank-Kasper polyhedra with a coordination number of 16 , so called Friauf polyhedra (see Fig. 3). Around the $4 c / 4 d$ position these polyhedra are spread up by anions on the $16 e$ and $4 a$ positions. Four of these Friauf polyhedra are arranged around the $B$-cation position forming a tetrahedral void centred by $4 b$ (Fig. 4). A Friauf polyhedron itself can be divided into 
Table 5b. Anisotropic displacement parameters $U_{i j}$ in $\AA^{2}$ with e.s.d.s in parentheses for the argyrodites of Type 2, 3 , and 4 .

\begin{tabular}{|c|c|c|c|c|c|c|}
\hline & $U_{11}$ & $U_{22}$ & $U_{33}$ & $U_{12}$ & $U_{13}$ & $U_{23}$ \\
\hline \multicolumn{7}{|l|}{ Type 2} \\
\hline \multicolumn{7}{|c|}{$\mathrm{Cu}_{7.49} \mathrm{SiS}_{5.49} \mathbf{I}_{0.51}$} \\
\hline $\mathrm{Cu} 1$ & $0.0184(5)$ & $0.062(1)$ & $U_{22}$ & 0 & 0 & $0.042(1)$ \\
\hline $\mathrm{Cu} 2$ & $0.074(2)$ & $0.068(2)$ & $U_{22}$ & $0.020(1)$ & $U_{12}$ & $0.045(2)$ \\
\hline $\mathrm{Cu} 3$ & $0.11(1)$ & $U_{11}$ & $U_{11}$ & $-0.048(5)$ & $U_{12}$ & $U_{12}$ \\
\hline $\mathrm{S} 1$ & $0.0086(2)$ & $U_{11}$ & $U_{11}$ & $-0.003(1)$ & $U_{12}$ & $U_{12}$ \\
\hline $\mathrm{S} 2$ & $0.0165(3)$ & $U_{11}$ & $U_{11}$ & 0 & 0 & 0 \\
\hline $\mathrm{I} 3 / \mathrm{S} 3$ & $0.0185(2)$ & $U_{11}$ & $U_{11}$ & 0 & 0 & 0 \\
\hline $\mathrm{Si}$ & $0.0063(3)$ & $U_{11}$ & $U_{11}$ & 0 & 0 & 0 \\
\hline \multicolumn{7}{|c|}{$\mathrm{Cu}_{6} \mathrm{PS}_{5} \mathrm{Cl}$} \\
\hline $\mathrm{Cu} 1$ & $0.0195(5)$ & $0.043(1)$ & $U_{22}$ & 0 & 0 & $0.024(1)$ \\
\hline $\mathrm{Cu} 2$ & $0.033(1)$ & $0.044(1)$ & $U_{22}$ & $-0.001(1)$ & $U_{12}$ & $0.021(1)$ \\
\hline $\mathrm{Cu} 3$ & $0.061(7)$ & $U_{11}$ & $U_{11}$ & $-0.025(4)$ & $U_{12}$ & $U_{12}$ \\
\hline $\mathrm{S} 1$ & $0.0127(2)$ & $U_{11}$ & $U_{11}$ & $-0.003(1)$ & $U_{12}$ & $U_{12}$ \\
\hline S2 & $0.0208(4)$ & $U_{11}$ & $U_{11}$ & 0 & 0 & 0 \\
\hline $\mathrm{Cl}$ & $0.0350(6)$ & $U_{11}$ & $U_{11}$ & 0 & 0 & 0 \\
\hline $\mathrm{P}$ & $0.0082(3)$ & $U_{11}$ & $U_{11}$ & 0 & 0 & 0 \\
\hline \multicolumn{7}{|l|}{ Type 3} \\
\hline \multicolumn{7}{|c|}{$\mathrm{Cu}_{7.82} \mathrm{SiS}_{5.82} \mathrm{Br}_{0.18}$} \\
\hline $\mathrm{Cu} 1$ & $0.0210(4)$ & $0.056(1)$ & $U_{22}$ & 0 & 0 & $0.035(1)$ \\
\hline $\mathrm{Cu} 2$ & $0.074(2)$ & $0.063(1)$ & $U_{22}$ & $0.033(2)$ & $U_{12}$ & $0.023(2)$ \\
\hline $\mathrm{Cu} 3$ & $0.13(1)$ & $0.056(2)$ & $U_{22}$ & $-0.030(3)$ & $U_{12}$ & $-0.014(2)$ \\
\hline S1 & $0.0122(2)$ & $U_{11}$ & $U_{11}$ & $-0.003(1)$ & $U_{12}$ & $U_{12}$ \\
\hline S2 & $0.0192(3)$ & $U_{11}$ & $U_{11}$ & 0 & 0 & 0 \\
\hline $\mathrm{Br} 3 / \mathrm{S} 3$ & $0.0317(5)$ & $U_{11}$ & $U_{11}$ & 0 & 0 & 0 \\
\hline $\mathrm{Si}$ & $0.0080(3)$ & $U_{11}$ & $U_{11}$ & 0 & 0 & 0 \\
\hline \multicolumn{7}{|c|}{$\mathrm{Cu}_{7.75} \mathrm{GeS}_{5.75} \mathrm{Br}_{0.25}$} \\
\hline $\mathrm{Cu} 1$ & $0.020(1)$ & $0.046(2)$ & $U_{22}$ & 0 & 0 & $0.022(3)$ \\
\hline $\mathrm{Cu} 2$ & $0.067(7)$ & $0.090(9)$ & $U_{22}$ & $0.040(5)$ & $U_{12}$ & $0.06(1)$ \\
\hline $\mathrm{Cu} 3$ & $0.17(2)$ & $0.041(3)$ & $U_{22}$ & $-0.037(6)$ & $U_{12}$ & $-0.004(4)$ \\
\hline S1 & $0.0145(4)$ & $U_{11}$ & $U_{11}$ & $-0.003(1)$ & $U_{12}$ & $U_{12}$ \\
\hline $\mathrm{S} 2$ & $0.0213(6)$ & $U_{11}$ & $U_{11}$ & 0 & 0 & 0 \\
\hline $\mathrm{Br} 3 / \mathrm{S} 3$ & $0.030(1)$ & $U_{11}$ & $U_{11}$ & 0 & 0 & 0 \\
\hline $\mathrm{Ge}$ & $0.0091(3)$ & $U_{11}$ & $U_{11}$ & 0 & 0 & 0 \\
\hline \multicolumn{7}{|l|}{ Type 4} \\
\hline \multicolumn{7}{|c|}{$\mathrm{Cu}_{7.52} \mathrm{GeSe}_{5.52} \mathbf{I}_{0.48}$} \\
\hline $\mathrm{Cu} 1$ & $0.029(1)$ & $0.076(3)$ & $U_{22}$ & 0 & $U_{12}$ & $0.049(3)$ \\
\hline $\mathrm{Cu} 2$ & $0.082(3)$ & $0.062(2)$ & $U_{22}$ & $-0.016(2)$ & $U_{12}$ & $0.019(2)$ \\
\hline $\mathrm{Cu} 3$ & $0.21(5)$ & $0.018(9)$ & $\mathrm{U}^{11}$ & 0 & $-0.12(5)$ & $U_{12}$ \\
\hline Se1 & $0.0168(2)$ & $U_{11}$ & $U_{11}$ & $-0.003(1)$ & $U_{12}$ & $U_{12}$ \\
\hline $\mathrm{Se} 2$ & $0.0333(4)$ & $U_{11}$ & $U_{11}$ & 0 & 0 & 0 \\
\hline $\mathrm{I} 3$ & $0.0306(3)$ & $U_{11}$ & $U_{11}$ & 0 & 0 & 0 \\
\hline $\mathrm{Se} 3$ & $0.0306(3)$ & $U_{11}$ & $U_{11}$ & 0 & 0 & 0 \\
\hline $\mathrm{Ge}$ & $0.0114(2)$ & $U_{11}$ & $U_{11}$ & 0 & 0 & 0 \\
\hline
\end{tabular}

sets of trigonal biyramids as shown in Fig. 5. These biyramids represent the smallest section of the rigid substructure necessary to analyse the distribution of the mobile $A$ cations in the argyrodites. The linear, three- and four-coordinated copper positions present in the argyrodites can be all localized relative to only one of the defined bipyramids (see Fig. 6a and 6b). The argyrodites under discussion can be separated into four different types according to the copper distribution and copper content. In general, the num- ber of copper positions increases with an increasing copper content as expected. The copper distribution of the group 15 argyrodites (according the $B$-cation), showing the lowest copper content per formula unit, can be described by a trigonally coordinated $24 \mathrm{~g}$ and a fourfold coordinated $48 a$ position. Those argyrodites and some of the group 14 ones with a high iodide content and therefore small copper contents relative to the maximum of 8 copper atoms per formula unit (e.g. $\mathrm{Cu}_{7} \mathrm{SiS}_{5} \mathrm{I}$ or 
Table 6a. Selected interatomic distances in $\AA$ and angles in ${ }^{\circ}$ with e.s.d.s in parentheses for the Type 1 argyrodites ( $B$-cation: group 15$)$.

\begin{tabular}{|c|c|c|c|}
\hline \multicolumn{4}{|l|}{ Type 1} \\
\hline \multicolumn{4}{|l|}{$\mathrm{Cu}_{6} \mathrm{PS}_{5} \mathrm{Br}$} \\
\hline $\mathrm{Cu} 1-\mathrm{Cu} 1$ & $3.111(1)$ & $\mathrm{S} 1-\mathrm{Cu} 1-\mathrm{S} 1$ & $102.30(5)$ \\
\hline $\mathrm{Cu} 1-\mathrm{Cu} 2$ & $0.812(8)$ & $\mathrm{S} 1-\mathrm{Cu} 1-\mathrm{S} 2$ & $128.85(2)$ \\
\hline $\mathrm{Cu} 1-\mathrm{S} 1$ & $2.2612(8)$ & & \\
\hline $\mathrm{Cu} 1-\mathrm{S} 2$ & $2.200(1)$ & & \\
\hline $\mathrm{Cu} 2-\mathrm{Cu} 2$ & $1.62(1)$ & $\mathrm{S} 1-\mathrm{Cu} 2-\mathrm{S} 1$ & $95.5(2)$ \\
\hline $\mathrm{Cu} 2-\mathrm{S} 1$ & $2.379(7)$ & $\mathrm{S} 1-\mathrm{Cu} 2-\mathrm{S} 2$ & $115.4(3)$ \\
\hline $\mathrm{Cu} 2-\mathrm{S} 2$ & $2.382(3)$ & $\mathrm{S} 1-\mathrm{Cu} 2-\mathrm{Br}$ & $107.3(3)$ \\
\hline $\mathrm{Cu} 2-\mathrm{Br}$ & $2.635(8)$ & $\mathrm{S} 2-\mathrm{Cu} 2-\mathrm{Br}$ & $114.1(2)$ \\
\hline $\mathrm{P}-\mathrm{S} 1$ & $2.0551(5)$ & $\mathrm{S} 1-\mathrm{P}-\mathrm{S} 1$ & $109.47(2)$ \\
\hline \multicolumn{4}{|l|}{$\mathrm{Cu}_{6} \mathbf{P S}_{5} \mathrm{I}$} \\
\hline $\mathrm{Cu} 1-\mathrm{Cu} 1$ & $3.136(2)$ & $\mathrm{S} 1-\mathrm{Cu} 1-\mathrm{S} 1$ & $102.98(7)$ \\
\hline $\mathrm{Cu} 1-\mathrm{Cu} 2$ & $0.68(1)$ & $\mathrm{S} 1-\mathrm{Cu} 1-\mathrm{S} 2$ & $128.51(3)$ \\
\hline $\mathrm{Cu} 1-\mathrm{S} 1$ & $2.274(1)$ & & \\
\hline $\mathrm{Cu} 1-\mathrm{S} 2$ & $2.218(2)$ & & \\
\hline $\mathrm{Cu} 2-\mathrm{Cu} 2$ & $1.36(2)$ & $\mathrm{S} 1-\mathrm{Cu} 2-\mathrm{S} 1$ & $98.4(3)$ \\
\hline $\mathrm{Cu} 2-\mathrm{S} 1$ & $2.35(1)$ & $\mathrm{S} 1-\mathrm{Cu} 2-\mathrm{S} 2$ & $118.5(6)$ \\
\hline $\mathrm{Cu} 2-\mathrm{S} 2$ & $2.358(7)$ & $\mathrm{S} 1-\mathrm{Cu} 2-\mathrm{I}$ & $104.5(5)$ \\
\hline $\mathrm{Cu} 2-\mathrm{I}$ & $2.78(1)$ & $\mathrm{S} 2-\mathrm{Cu} 2-\mathrm{I}$ & $110.7(4)$ \\
\hline $\mathrm{P}-\mathrm{S} 1$ & $2.0571(4)$ & $\mathrm{S} 1-\mathrm{P}-\mathrm{S} 1$ & $109.47(2)$ \\
\hline \multicolumn{4}{|c|}{$\mathrm{Cu}_{6} \mathrm{AsS}_{5} \mathrm{Br}$} \\
\hline $\mathrm{Cu} 1-\mathrm{Cu} 1$ & $3.1365(7)$ & $\mathrm{S} 1-\mathrm{Cu} 1-\mathrm{S} 1$ & $97.83(3)$ \\
\hline $\mathrm{Cu} 1-\mathrm{Cu} 2$ & $0.825(7)$ & $\mathrm{S} 1-\mathrm{Cu} 1-\mathrm{S} 2$ & $131.08(2)$ \\
\hline $\mathrm{Cu} 1-\mathrm{S} 1$ & $2.2731(6)$ & & \\
\hline $\mathrm{Cu} 1-\mathrm{S} 2$ & $2.2178(7)$ & & \\
\hline $\mathrm{Cu} 2-\mathrm{Cu} 2$ & $1.65(1)$ & $\mathrm{S} 1-\mathrm{Cu} 2-\mathrm{S} 1$ & $92.0(1)$ \\
\hline $\mathrm{Cu} 2-\mathrm{S} 1$ & $2.383(6)$ & $\mathrm{S} 1-\mathrm{Cu} 2-\mathrm{S} 2$ & $116.7(3)$ \\
\hline $\mathrm{Cu} 2-\mathrm{S} 2$ & $2.419(3)$ & $\mathrm{S} 1-\mathrm{Cu} 2-\mathrm{Br}$ & $107.6(2)$ \\
\hline $\mathrm{Cu} 2-\mathrm{Br}$ & $2.665(7)$ & $\mathrm{S} 2-\mathrm{Cu} 2-\mathrm{Br}$ & $113.9(2)$ \\
\hline As-S1 & $2.1651(3)$ & $\mathrm{S} 1-\mathrm{As}-\mathrm{S} 1$ & $109.47(1)$ \\
\hline \multicolumn{4}{|l|}{$\mathrm{Cu}_{6} \mathrm{AsS}_{5} \mathrm{I}$} \\
\hline $\mathrm{Cu} 1-\mathrm{Cu} 1$ & $3.161(2)$ & $\mathrm{S} 1-\mathrm{Cu} 1-\mathrm{S} 1$ & $98.27(7)$ \\
\hline $\mathrm{Cu} 1-\mathrm{Cu} 2$ & $0.70(2)$ & $\mathrm{S} 1-\mathrm{Cu} 1-\mathrm{S} 2$ & $130.87(4)$ \\
\hline $\mathrm{Cu} 1-\mathrm{S} 1$ & $2.283(1)$ & & \\
\hline $\mathrm{Cu} 1-\mathrm{S} 2$ & $2.236(2)$ & & \\
\hline $\mathrm{Cu} 2-\mathrm{Cu} 2$ & $1.40(2)$ & $\mathrm{S} 1-\mathrm{Cu} 2-\mathrm{S} 1$ & $94.2(4)$ \\
\hline $\mathrm{Cu} 2-\mathrm{S} 1$ & $2.36(1)$ & $\mathrm{S} 1-\mathrm{Cu} 2-\mathrm{S} 2$ & $119.9(7)$ \\
\hline $\mathrm{Cu} 2-\mathrm{S} 2$ & $2.39(1)$ & $\mathrm{S} 1-\mathrm{Cu} 2-\mathrm{I}$ & $104.7(5)$ \\
\hline $\mathrm{Cu} 2-\mathrm{I}$ & 2.81(2) & $\mathrm{S} 2-\mathrm{Cu} 2-\mathrm{I}$ & $110.9(5)$ \\
\hline As-S1 & $2.1723(5)$ & $\mathrm{S} 1-\mathrm{As}-\mathrm{S} 1$ & $109.47(2)$ \\
\hline
\end{tabular}

$\left.\mathrm{Cu}_{7.44} \mathrm{SiSi}_{5.44} \mathrm{I}_{0.56}\right)$ are representing the Type 1 structure argyrodites.

Besides the type 1 argyrodites a linear coordinated $16 e$ position was observed for the Type 2 argyrodites. $\mathrm{Cu}_{6} \mathrm{PS}_{5} \mathrm{Cl}$ and $\mathrm{Cu}_{7.49} \mathrm{SiS}_{5.49} \mathrm{I}_{0.51}$ are representing the type 2 argyrodites. Surprisingly, $\mathrm{Cu}_{6} \mathrm{PS}_{5} \mathrm{Cl}$ belongs to the Type 2 argyrodites in contrast to the heavier homologues. This finding fits well to the unexpected trend of the ionic conductivities of $\mathrm{Cu}_{6} \mathrm{PS}_{5} \mathrm{X}(\mathrm{X}=\mathrm{Cl}, \mathrm{Br}, \mathrm{I})$ reported by Kuhs
Table 6b. Selected interatomic distances in $\AA$ and angles in ${ }^{\circ}$ with e.s.d.s in parentheses for the Type 1 argyrodites (B-cation: group 14). The maxima of electron density, the so-called mode positions (suffix $d$ ), were used for distance and angle calculations in case of a nonharmonic development of the displacement parameters.

\begin{tabular}{|c|c|c|c|}
\hline \multicolumn{4}{|l|}{ Type 1} \\
\hline \multicolumn{4}{|l|}{$\mathrm{Cu}_{7} \mathrm{SiS}_{5} \mathrm{I}$} \\
\hline $\mathrm{Cu} 1-\mathrm{Cu} 1$ & $3.189(1)$ & & \\
\hline $\mathrm{Cu} 1-\mathrm{Cu} 2_{d}$ & $0.8182(2)$ & & \\
\hline $\mathrm{Cu} 1-\mathrm{S} 1$ & $2.304(1)$ & $\mathrm{S} 1-\mathrm{Cu} 1-\mathrm{S} 1$ & $101.63(5)$ \\
\hline $\mathrm{Cu} 1-\mathrm{I} 2 / \mathrm{S} 2$ & $2.255(1)$ & $\mathrm{S} 1-\mathrm{Cu} 1-\mathrm{I} 2 / \mathrm{S} 2$ & $129.18(3)$ \\
\hline $\mathrm{Cu} 2_{d}-\mathrm{Cu} 2_{d}$ & 1.6176 & $\mathrm{~S} 1-\mathrm{Cu} 2_{d}-\mathrm{S} 1$ & $97.78(2)$ \\
\hline $\mathrm{Cu} 2{ }_{d}-\mathrm{S} 1$ & $2.3702(6)$ & $\mathrm{S} 1-\mathrm{Cu} 2{ }_{d}-\mathrm{I} 2 / \mathrm{S} 2$ & $114.98(1)$ \\
\hline $\mathrm{Cu} 2{ }_{d}-\mathrm{I} 2 / \mathrm{S} 2$ & 2.5119 & $\mathrm{~S} 1-\mathrm{Cu} 2{ }_{d}-\mathrm{I} 3 / \mathrm{S} 3$ & $108.57(1)$ \\
\hline $\mathrm{Cu} 2_{d}-\mathrm{I} 3 / \mathrm{S} 3$ & 2.7099 & $\mathrm{I} 2 / \mathrm{S} 2-\mathrm{Cu} 2_{d}-\mathrm{I} 3 / \mathrm{S} 3$ & 111.076 \\
\hline $\mathrm{Si}-\mathrm{S} 1$ & $2.1197(6)$ & $\mathrm{S} 1-\mathrm{Si}-\mathrm{S} 1$ & $109.47(2)$ \\
\hline \multicolumn{4}{|l|}{$\mathrm{Cu}_{7} \mathrm{GeS}_{5} \mathbf{I}$} \\
\hline $\mathrm{Cu} 1-\mathrm{Cu} 1$ & $3.193(1)$ & $\mathrm{S} 1-\mathrm{Cu} 1-\mathrm{S} 1$ & $98.25(5)$ \\
\hline $\mathrm{Cu} 1-\mathrm{Cu} 2_{d}$ & $0.8362(2)$ & $\mathrm{S} 1-\mathrm{Cu} 1-\mathrm{I} 2 / \mathrm{S} 2$ & $130.87(3)$ \\
\hline $\mathrm{Cu} 1-\mathrm{S} 1$ & $2.314(1)$ & & \\
\hline $\mathrm{Cu} 1-\mathrm{I} 2 / \mathrm{S} 2$ & $2.258(1)$ & & \\
\hline $\mathrm{Cu} 2_{d}-\mathrm{Cu} 2_{d}$ & 1.652 & $\mathrm{~S} 1-\mathrm{Cu} 2{ }_{d}-\mathrm{S} 1$ & $94.71(2)$ \\
\hline $\mathrm{Cu} 2{ }_{d}-\mathrm{S} 1$ & $2.3786(7)$ & $\mathrm{S} 1-\mathrm{Cu} 2{ }_{d}-\mathrm{I} 2 / \mathrm{S} 2$ & $115.86(2)$ \\
\hline $\mathrm{Cu} 2{ }_{d}-\mathrm{I} 2 / \mathrm{S} 2$ & 2.5271 & $\mathrm{~S} 1-\mathrm{Cu} 2{ }_{d}-\mathrm{I} 3 / \mathrm{S} 3$ & $108.79(2)$ \\
\hline $\mathrm{Cu} 2{ }_{d}-\mathrm{I} 3 / \mathrm{S} 3$ & 2.7184 & $\mathrm{I} 2 / \mathrm{S} 2-\mathrm{Cu} 2_{d}-\mathrm{I} 3 / \mathrm{S} 3$ & 111.528 \\
\hline $\mathrm{Ge}-\mathrm{S} 1$ & $2.1951(7)$ & $\mathrm{S} 1-\mathrm{Ge}-\mathrm{S} 1$ & $109.47(3)$ \\
\hline \multicolumn{4}{|c|}{$\mathrm{Cu}_{7.44} \mathrm{SiSe}_{5.44} \mathbf{I}_{0.56}$} \\
\hline $\mathrm{Cu} 1-\mathrm{Se} 1$ & $2.408(2)$ & $\mathrm{Se} 1-\mathrm{Cu} 1-\mathrm{Se} 1$ & $96.80(9)$ \\
\hline $\mathrm{Cu} 1-\mathrm{Se} 2$ & $2.21(2)$ & $\mathrm{Se} 1-\mathrm{Cu} 1-\mathrm{Se} 2$ & $131.6(4)$ \\
\hline $\mathrm{Cu} 1-\mathrm{Se} 2$ & $2.33(2)$ & $\mathrm{Se} 1-\mathrm{Cu} 1-\mathrm{Se} 2$ & $129.6(4)$ \\
\hline $\mathrm{Cu} 1-\mathrm{Cu} 2_{d}$ & $1.0056(3)$ & $\mathrm{Se} 1-\mathrm{Cu} 1-\mathrm{Se} 2$ & $133.6(4)$ \\
\hline $\mathrm{Cu} 2_{d}-\mathrm{Se} 1$ & $2.5247(8)$ & $\mathrm{Se} 1-\mathrm{Cu} 2_{d}-\mathrm{Se} 1$ & $90.99(3)$ \\
\hline $\mathrm{Cu} 2_{d}-\mathrm{Se} 2$ & $2.58(2)$ & $\mathrm{Se} 1-\mathrm{Cu} 2_{d}-\mathrm{Se} 2$ & 111.2(3) \\
\hline $\mathrm{Cu} 2_{d}-\mathrm{Se} 2$ & $2.66(2)$ & $\mathrm{Se} 1-\mathrm{Cu} 2_{d}-\mathrm{Se} 2$ & $111.5(3)$ \\
\hline $\mathrm{Cu} 2_{d}-\mathrm{I} 3 / \mathrm{Se} 3$ & 2.6451 & $\mathrm{Se} 1-\mathrm{Cu} 2_{d}-\mathrm{Se} 2$ & $113.4(4)$ \\
\hline \multirow[t]{5}{*}{$\mathrm{Cu} 2_{d}-\mathrm{Cu} 2_{d}$} & 1.9927 & $\mathrm{Se} 1-\mathrm{Cu} 2{ }_{d}-\mathrm{Se} 2$ & 114.3(3) \\
\hline & & $\mathrm{Se} 1-\mathrm{Cu} 2{ }_{d}-\mathrm{I} 3 / \mathrm{Se} 3$ & $110.97(2)$ \\
\hline & & $\mathrm{Se} 2-\mathrm{Cu} 2{ }_{d}-\mathrm{I} 3 / \mathrm{Se} 3$ & $118.2(3)$ \\
\hline & & $\mathrm{Se} 2-\mathrm{Cu} 2{ }_{d}-\mathrm{I} 3 / \mathrm{Se} 3$ & $115.6(3)$ \\
\hline & & $\mathrm{Se} 2-\mathrm{Cu} 2{ }_{d}-\mathrm{I} 3 / \mathrm{Se} 3$ & $114.8(4)$ \\
\hline $\mathrm{Si}-\mathrm{Se} 1$ & $2.2484(8)$ & $\mathrm{Se} 1-\mathrm{Si}-\mathrm{Se} 1$ & $109.47(3)$ \\
\hline
\end{tabular}

el al. (1979). Kuhs found the highest ion conductivity for $\mathrm{Cu}_{6} \mathrm{PS}_{5} \mathrm{Cl}$ followed by $\mathrm{Cu}_{6} \mathrm{PS}_{5} \mathrm{I}$ and $\mathrm{Cu}_{6} \mathrm{PS}_{5} \mathrm{Br}$. No proper reason was given for this finding. A significant higher copper mobility can be expected for $\mathrm{Cu}_{6} \mathrm{PS}_{5} \mathrm{Cl}$ than for the other two homologues based on the differences in copper coordination and distribution.

\section{Joint probability density function (jpdf) analysis}

After a non-harmonic refinement of the $A$-cation substructure a joint probability density function analysis was applied to identify possible diffusion pathways for the mobile ions and to highlight the differences in copper 
Table 6c. Selected interatomic distances in $\AA$ and angles in ${ }^{\circ}$ with e.s.d.s in parentheses for the Type 2 argyrodites. The maxima of electron density, the so-called mode positions ( $\operatorname{suffix} d$ ) were used for distance and angle calculations in case of a non-harmonic development of displacement parameters.

\begin{tabular}{|c|c|c|c|}
\hline \multicolumn{4}{|l|}{ Type 2} \\
\hline \multicolumn{4}{|c|}{$\mathrm{Cu}_{7.49} \mathrm{SiS}_{5.49} \mathbf{I}_{0.51}$} \\
\hline $\mathrm{Cu} 1-\mathrm{Cu} 1$ & $3.157(1)$ & $\mathrm{S} 1-\mathrm{Cu} 1-\mathrm{S} 1$ & $100.97(6)$ \\
\hline$-\mathrm{Cu} 3$ & $2.036(6)$ & $\mathrm{S} 1-\mathrm{Cu} 1-\mathrm{S} 2$ & $129.51(3)$ \\
\hline$-\mathrm{S} 1$ & $2.298(1)$ & $\mathrm{S} 1-\mathrm{Cu} 1-\mathrm{S} 1$ & $100.97(6)$ \\
\hline$-\mathrm{S} 2$ & $2.233(1)$ & $\mathrm{S} 1-\mathrm{Cu} 1-\mathrm{S} 2$ & $129.51(3)$ \\
\hline$-\mathrm{Cu} 2_{\mathrm{d}}$ & $0.8336(2)$ & & \\
\hline $\mathrm{Cu} 2_{d}-\mathrm{Cu} 2_{d}$ & 1.649 & $\mathrm{~S} 1-\mathrm{Cu} 2_{d}-\mathrm{S} 1$ & $96.88(2)$ \\
\hline$-\mathrm{Cu} 3$ & $1.457(6)$ & $\mathrm{S} 1-\mathrm{Cu} 2_{d}-\mathrm{S} 2$ & $114.73(1)$ \\
\hline$-\mathrm{S} 1$ & $2.3693(5)$ & $\mathrm{S} 1-\mathrm{Cu} 2_{d}-\mathrm{I} 3 / \mathrm{S} 3$ & $108.83(1)$ \\
\hline$-\mathrm{S} 2$ & 2.496 & $\mathrm{~S} 2-\mathrm{Cu} 2_{d}-\mathrm{I} 3 / \mathrm{S} 3$ & 111.803 \\
\hline$-\mathrm{I} 3 / \mathrm{S} 3$ & 2.677 & & \\
\hline $\mathrm{Cu} 3-\mathrm{S} 2$ & $2.197(6)$ & $\mathrm{S} 2-\mathrm{Cu} 3-\mathrm{I} 3 / \mathrm{S} 3$ & 180 \\
\hline$-\mathrm{I} 3 / \mathrm{S} 3$ & $2.089(6)$ & & \\
\hline $\mathrm{Si}-\mathrm{S} 1$ & $2.1141(5)$ & $\mathrm{S} 1-\mathrm{Si}-\mathrm{S} 1$ & $109.47(2)$ \\
\hline \multicolumn{4}{|l|}{$\mathrm{Cu}_{6} \mathrm{PS}_{5} \mathrm{Cl}$} \\
\hline $\mathrm{Cu} 1-\mathrm{Cu} 1$ & $3.087(1)$ & $\mathrm{S} 1-\mathrm{Cu} 1-\mathrm{S} 1$ & $101.78(5)$ \\
\hline $\mathrm{Cu} 1-\mathrm{Cu} 2_{d}$ & $0.8961(1)$ & $\mathrm{S} 1-\mathrm{Cu} 1-\mathrm{S} 2$ & $129.11(3)$ \\
\hline $\mathrm{Cu} 1-\mathrm{Cu} 3$ & $2.037(6)$ & & \\
\hline $\mathrm{Cu} 1-\mathrm{S} 1$ & $2.2540(9)$ & & \\
\hline $\mathrm{Cu} 1-\mathrm{S} 2$ & $2.183(1)$ & & \\
\hline $\mathrm{Cu} 2_{d}-\mathrm{Cu} 2_{d}$ & 1.788 & $\mathrm{~S} 1-\mathrm{Cu} 2_{d}-\mathrm{S} 1$ & $94.02(2)$ \\
\hline $\mathrm{Cu} 2_{d}-\mathrm{S} 1$ & $2.3909(6)$ & $\mathrm{S} 1-\mathrm{Cu} 2{ }_{d}-\mathrm{S} 2$ & 113.01(1) \\
\hline $\mathrm{Cu} 2_{d}-\mathrm{S} 2$ & 2.4133 & $\mathrm{~S} 1-\mathrm{Cu} 2_{d}-\mathrm{Cl}$ & $109.44(1)$ \\
\hline $\mathrm{Cu} 2_{d}-\mathrm{Cl}$ & 2.536 & $\mathrm{~S} 2-\mathrm{Cu} 2{ }_{d}-\mathrm{Cl}$ & 115.799 \\
\hline $\mathrm{Cu} 3-\mathrm{Cu} 3$ & $3.178(9)$ & $\mathrm{S} 2-\mathrm{Cu} 3-\mathrm{Cl}$ & 180 \\
\hline $\mathrm{Cu} 3-\mathrm{S} 2$ & $2.247(6)$ & & \\
\hline $\mathrm{Cu} 3-\mathrm{Cl}$ & $1.946(6)$ & & \\
\hline $\mathrm{P}-\mathrm{S} 1$ & $2.0507(6)$ & $\mathrm{S} 1-\mathrm{P}-\mathrm{S} 1$ & $109.47(2)$ \\
\hline
\end{tabular}

coordination for the argyrodites. The Type 1 and Type 2 argyrodites can be differentiated into subgroups after the jpdf analysis.

Type 1 argyrodites can be divided into two subgroups related to the PSE group number of the $B$-cation and the resulting copper content of the argyrodites. The higher

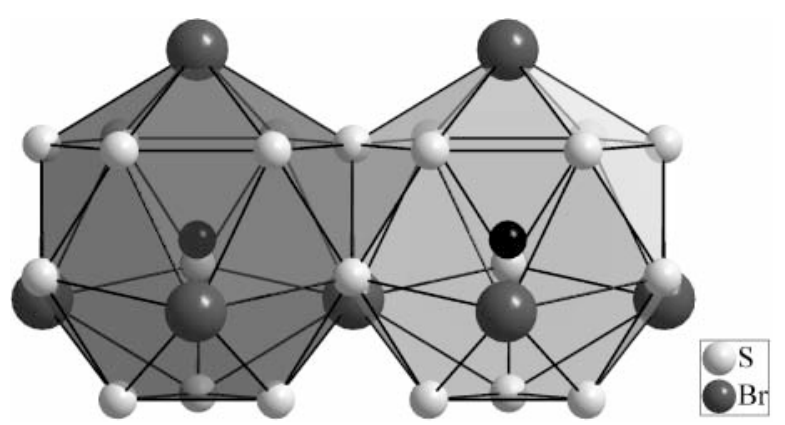

Fig. 3. Face sharing Frank-Kasper polyhedra around a $4 c / 4 d$ position (small black spheres) in cubic argyrodites illustrated for $\mathrm{Cu}_{6} \mathrm{PS}_{5} \mathrm{Br}$. The polyhedra are connected via $S_{2} X$ faces.
Table 6d. Selected interatomic distances in $\AA$ and angles in ${ }^{\circ}$ with e.s.d.s in parentheses for the Type 3 and Type 4 argyrodites. The maxima of electron density, the so-called mode positions (suffix d), were used for distance and angle calculations in case of a non-harmonic development of displacement parameters.

\begin{tabular}{|c|c|c|c|}
\hline Type 3 & & & \\
\hline $\mathrm{Cu}_{7.82} \mathrm{SiS}_{5.82} \mathrm{~B}$ & & & \\
\hline $\mathrm{Cu} 1-\mathrm{Cu} 1$ & $3.1360(9)$ & $\mathrm{S} 1-\mathrm{Cu} 1-\mathrm{S} 1$ & $100.62(4)$ \\
\hline$-\mathrm{Cu} 2_{d}$ & 0.9981 & $\mathrm{~S} 1-\mathrm{Cu} 1-\mathrm{S} 2$ & $129.69(2)$ \\
\hline$-\mathrm{Cu} 3$ & $1.46(1)$ & & \\
\hline$-\mathrm{S} 1$ & $2.2831(8)$ & & \\
\hline$-\mathrm{S} 2$ & $2.2175(9)$ & & \\
\hline $\mathrm{Cu} 2_{d}-\mathrm{Cu} 2_{d}$ & 1.9946 & $\mathrm{~S} 1-\mathrm{Cu} 2_{d}-\mathrm{S} 1$ & $90.73(2)$ \\
\hline$-\mathrm{Cu} 3$ & $0.64(2)$ & $\mathrm{S} 1-\mathrm{Cu} 2_{d}-\mathrm{S} 2$ & $111.25(1)$ \\
\hline$-\mathrm{S} 1$ & $2.4689(5)$ & $\mathrm{S} 1-\mathrm{Cu} 2_{d}-\mathrm{Br} 3 / \mathrm{S} 3$ & $110.84(1)$ \\
\hline$-\mathrm{S} 2$ & 2.4669 & $\mathrm{~S} 2-\mathrm{Cu} 2_{d}-\mathrm{Br} 3 / \mathrm{S} 3$ & 118.517 \\
\hline$-\mathrm{Br} 3 / \mathrm{S} 3$ & 2.4884 & & \\
\hline$-\mathrm{Cu} 3$ & $1.66(2)$ & $\mathrm{S} 1-\mathrm{Cu} 3-\mathrm{S} 1$ & $95.7(4)$ \\
\hline $\mathrm{Cu} 3-\mathrm{S} 1$ & $2.37(1)$ & $\mathrm{S} 1-\mathrm{Cu} 3-\mathrm{S} 2$ & $95.2(4)$ \\
\hline$-\mathrm{S} 2$ & $3.11(2)$ & $\mathrm{S} 1-\mathrm{Cu} 3-\mathrm{Br} 3 / \mathrm{S} 3$ & $127.8(4)$ \\
\hline$-\mathrm{Br} 3 / \mathrm{S} 3$ & $2.174(7)$ & $\mathrm{S} 2-\mathrm{Cu} 3-\mathrm{Br} 3 / \mathrm{S} 3$ & 106.2(7) \\
\hline $\mathrm{Si}-\mathrm{S} 1$ & $2.1072(5)$ & $\mathrm{S} 1-\mathrm{Si}-\mathrm{S} 1$ & $109.47(2)$ \\
\hline $\mathrm{Cu}_{7.75} \mathrm{GeS}_{5.75}$ & & & \\
\hline $\mathrm{Cu} 1-\mathrm{Cu} 1$ & $3.155(3)$ & $\mathrm{S} 1-\mathrm{Cu} 1-\mathrm{S} 1$ & $97.5(1)$ \\
\hline $\mathrm{Cu} 1-\mathrm{Cu} 2_{d}$ & $0.9157(1)$ & $\mathrm{S} 1-\mathrm{Cu} 1-\mathrm{S} 2$ & $131.26(5)$ \\
\hline $\mathrm{Cu} 1-\mathrm{Cu} 3$ & $1.46(2)$ & & \\
\hline $\mathrm{Cu} 1-\mathrm{S} 1$ & $2.300(2)$ & & \\
\hline $\mathrm{Cu} 1-\mathrm{S} 2$ & $2.231(3)$ & & \\
\hline $\mathrm{Cu} 2_{d}-\mathrm{Cu} 3$ & $0.72(3)$ & $\mathrm{S} 1-\mathrm{Cu} 2_{d}-\mathrm{S} 1$ & $89.49(3)$ \\
\hline $\mathrm{Cu} 2_{d}-\mathrm{S} 1$ & $2.456(1)$ & $\mathrm{S} 1-\mathrm{Cu} 2_{d}-\mathrm{S} 2$ & $114.89(2)$ \\
\hline $\mathrm{Cu} 2_{d}-\mathrm{S} 2$ & 2.4404 & $\mathrm{~S} 1-\mathrm{Cu} 2_{d}-\mathrm{Br} 3 / \mathrm{S} 3$ & $108.64(2)$ \\
\hline $\mathrm{Cu} 2_{d}-\mathrm{Br} 3 / \mathrm{S} 3$ & 2.6087 & $\mathrm{~S} 2-\mathrm{Cu} 2_{d}-\mathrm{Br} 3 / \mathrm{S} 3$ & 116.92 \\
\hline $\mathrm{Cu} 3-\mathrm{Cu} 3$ & $1.67(3)$ & $\mathrm{S} 1-\mathrm{Cu} 3-\mathrm{S} 1$ & $94.4(7)$ \\
\hline $\mathrm{Cu} 3-\mathrm{S} 1$ & $2.36(2)$ & $\mathrm{S} 1-\mathrm{Cu} 3-\mathrm{S} 2$ & $96.2(6)$ \\
\hline $\mathrm{Cu} 3-\mathrm{S} 2$ & $3.14(3)$ & $\mathrm{S} 1-\mathrm{Cu} 3-\mathrm{Br} 3 / \mathrm{S} 3$ & $128.1(6)$ \\
\hline $\mathrm{Cu} 3-\mathrm{Br} 3 / \mathrm{S} 3$ & $2.22(1)$ & $\mathrm{S} 2-\mathrm{Cu} 3-\mathrm{Br} 3 / 3$ & $105(1)$ \\
\hline $\mathrm{Ge}-\mathrm{S} 1$ & $2.187(1)$ & $\mathrm{S} 1-\mathrm{Ge}-\mathrm{S} 1$ & $109.47(4)$ \\
\hline
\end{tabular}

Type 4

$\mathrm{Cu}_{7.52} \mathrm{GeSe}_{5.52} \mathrm{I}_{0.48}$

$\begin{array}{llll}\mathrm{Cu} 1-\mathrm{Cu} 2_{d} & 0.9990(3) & \mathrm{Se} 1-\mathrm{Cu} 1-\mathrm{Se} 1 & 94.66(9) \\ \mathrm{Cu} 1-\mathrm{Cu} 3 & 2.663(4) & \mathrm{Se} 1-\mathrm{Cu} 1-\mathrm{Se} 2 & 132.67(5) \\ \mathrm{Cu} 1-\mathrm{Se} 1 & 2.420(2) & & \\ \mathrm{Cu} 1-\mathrm{Se} 2 & 2.292(3) & & \\ \mathrm{Cu} 2_{d}-\mathrm{Cu} 2_{d} & 1.9817 & \mathrm{Se} 1-\mathrm{Cu} 2_{d}-\mathrm{Se} 1 & 89.07(1) \\ \mathrm{Cu} 2_{d}-\mathrm{Cu} 3 & 1.911(2) & \mathrm{Se} 1-\mathrm{Cu} 2_{d}-\mathrm{Se} 2 & 113.81(1) \\ \mathrm{Cu} 2_{d}-\mathrm{Se} 1 & 2.5366(5) & \mathrm{Se} 1-\mathrm{Cu} 2_{d}-\mathrm{I} 3 / \mathrm{Se} 3 & 110.54(1) \\ \mathrm{Cu} 2_{d}-\mathrm{Se} 2 & 2.6146 & \mathrm{Se} 2-\mathrm{Cu} 2_{d}-\mathrm{I} 3 / \mathrm{Se} 3 & 116.017 \\ \mathrm{Cu} 2{ }_{d}-\mathrm{I} 3 / \mathrm{Se} 3 & 2.6847 & & \\ \mathrm{Cu} 3-\mathrm{Cu} 3 & 2.94(2) & \mathrm{Se} 1-\mathrm{Cu} 3-\mathrm{Se} 1 & 93.7(7) \\ \mathrm{Cu} 3-\mathrm{Se} 1 & 2.59(1) & \mathrm{Se} 1-\mathrm{Cu} 3-\mathrm{I} 3 / \mathrm{Se} 3 & 133.1(3) \\ \mathrm{Cu} 3-\mathrm{I} 3 / \mathrm{Se} 3 & 2.08(2) & & \\ \mathrm{Ge}-\mathrm{Se} 1 & 2.3158(5) & \mathrm{Se} 1-\mathrm{Ge}-\mathrm{Se} 1 & 109.47(2)\end{array}$




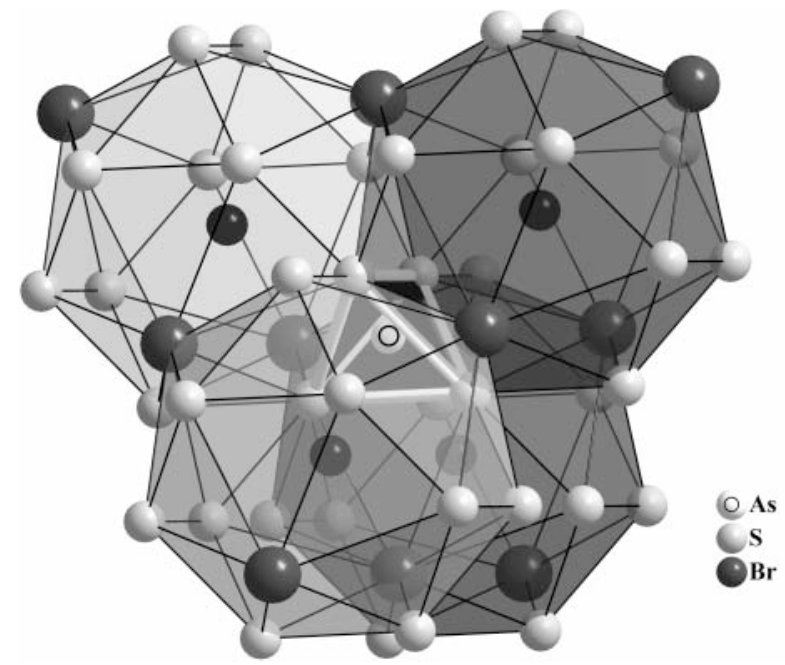

Fig. 4. Coordination of the $4 b$ position ( $B$-cation) by four Friauf polyhedra illustrated for $\mathrm{Cu}_{6} \mathrm{AsS}_{5} \mathrm{Br}$.

copper content of the group 14 argyrodites causes an increasing non harmonicity of the displacement parameters and an increased tendency to occupy $[3+1]$ coordinated positions (see Fig. 6a) compared to the group 15 members.

Copper in $\mathrm{Cu}_{7.49} \mathrm{SiS}_{5.49} \mathrm{I}_{0.51}$ (Type 2 group) tends to occupy tetrahedrally, trigonally and linearly coordinated positions within the above mentioned double tetrahedra.

Three diffusion pathways can be estimated for all four types from the shape of the corresponding jpdf. In Fig. 7 pathway 1 to 3 is illustrated by the two examples $\mathrm{Cu}_{7.49} \mathrm{SiS}_{5.49} \mathrm{I}_{0.51}$ (Type 2) and $\mathrm{Cu}_{7.52} \mathrm{GeSe}_{5.52} \mathrm{I}_{0.48}$ (Type 4).

Pathway 1 heading through a double tetrahedron face spread up by two $16 e$ and the $4 a$ position is the most favourable pathway of the Type 1 argyrodites, at least for the group 14 members. No significant preference can be observed for the group 15 members.

Type 2 argyrodites are supposed to be dominated by a copper diffusion along pathway $1\left(\mathrm{Cu}_{7.49} \mathrm{SiS}_{5.49} \mathrm{I}_{0.51}\right)$ or pathway $2\left(\mathrm{Cu}_{6} \mathrm{PS}_{5} \mathrm{Cl}\right)$.

No significant preference of pathway 1 or pathway 2 can be observed for Type 3 argyrodites.

A third pathway was found for the Type 4 argyrodite $\mathrm{Cu}_{7.52} \mathrm{GeSe}_{5.52} \mathrm{I}_{0.48}$. Pathway 3 heading through the face of

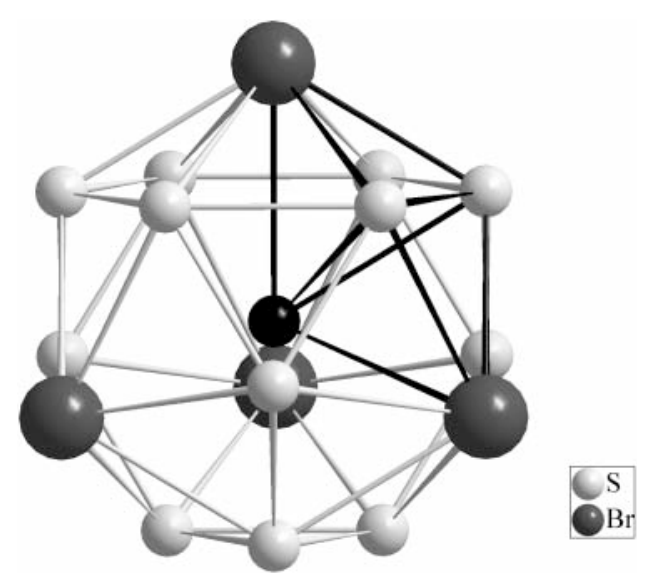

Fig. 5. Friauf polyhedra around $4 c / 4 d$ (black sphere) illustrated for $\mathrm{Cu}_{6} \mathrm{AsS}_{5} \mathrm{Br}$. Each Friauf polyhedron can be separated into 12 double tetrahedra.

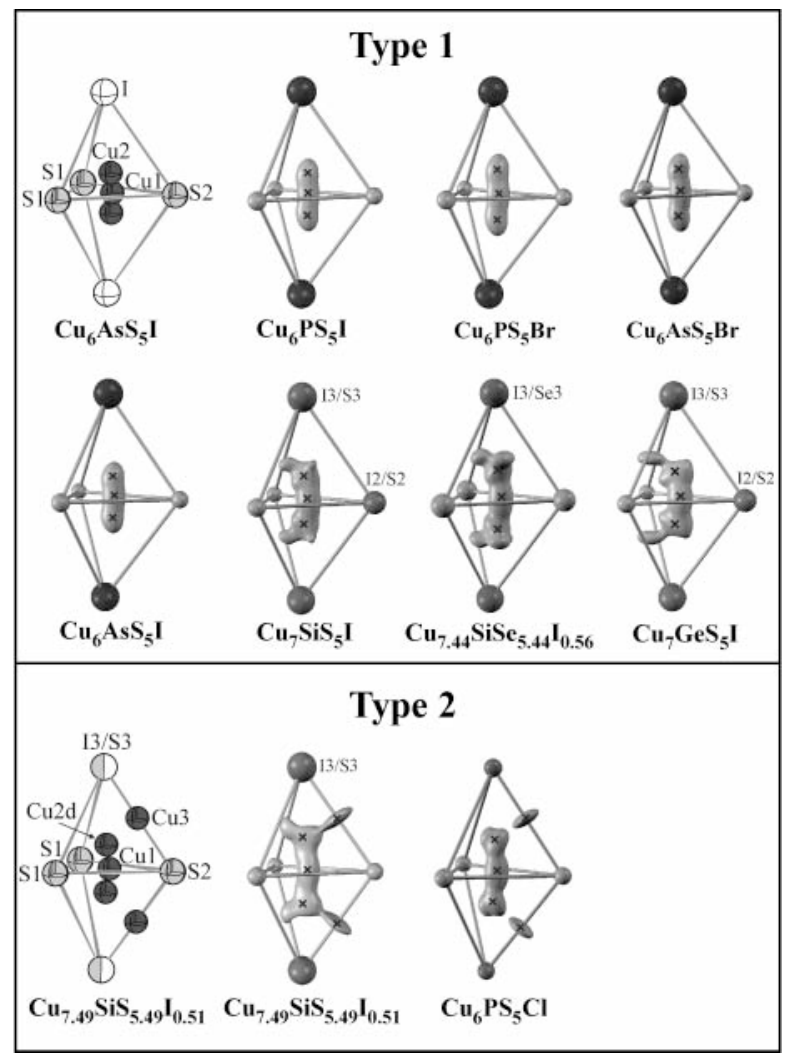

(a)

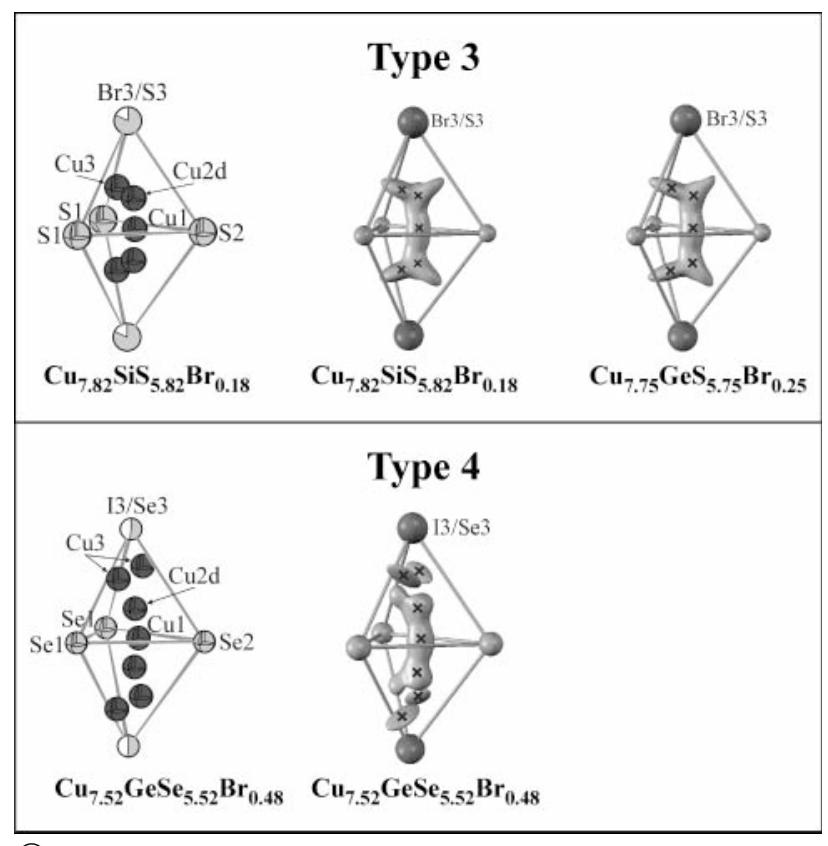

(b)

Fig. 6. (a) A-cation substructure of Type1 and Type 2 copper argyrodites. Type 1: trigonal coordinated $\mathrm{Cu} 1$ on $24 \mathrm{~g}$ and tetrahedrally coordinated $\mathrm{Cu} 2$ on $48 h$. Type 2: One additional two-coordinate $\mathrm{Cu} 3$ on $16 e$. Jpdf plots of the respective copper distribution illustrate the difference in copper coordination for the different argyrodites. Refined copper positions are marked by an $x$. (b) $A$-cation substructure of the Type 3 and Type 4 copper argyrodites. Type 3: One additional $[3+1]$ coordinated $\mathrm{Cu} 3$ besides the Type 1 positions $\mathrm{Cu} 1$ and $\mathrm{Cu} 2$ on 48h. Type 4: One additional $[3+1]$ coordinated $\mathrm{Cu} 3$ besides the Type 1 positions $\mathrm{Cu} 1$ and $\mathrm{Cu} 2$ on $24 f$. Jpdf plots of the respective copper distribution illustrate the difference in copper coordination for the different argyrodites. Refined copper positions are marked. 

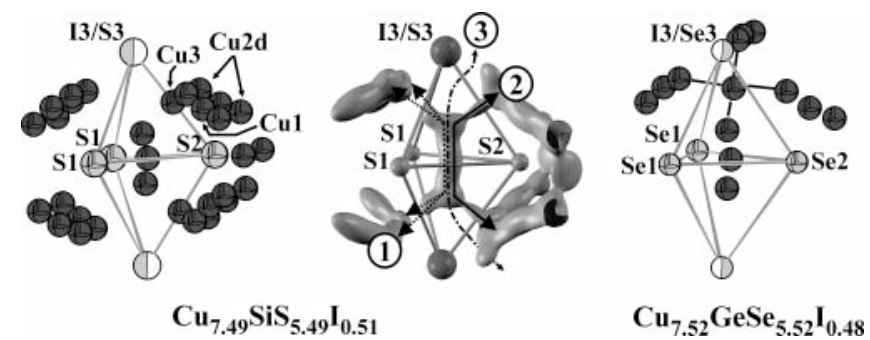

Fig. 7. Three copper diffusion pathways realized for the Type 1-4 argyrodites estimated from the shape of the jpdf. Examples are $\mathrm{Cu}_{7.49} \mathrm{SiS}_{5.49} \mathrm{I}_{0.51}$ and $\mathrm{Cu}_{7.52} \mathrm{GeSe}_{5.52} \mathrm{I}_{0.48}$.

a double tetrahedron which is spread up by a $16 e, 4 d$ and a $4 a$ position completes the set of different pathways realized by the copper argyrodites.

\section{One particle potential (opp) analysis}

In order to identify the favourite diffusion pathways and to classify the present argyrodites in terms of their potential ionic conductivity we performed one particle potential (opp) analysis along each pathway. Opps can be derived from the related jpdfs (Kuhs, 1992; Bachmann and Schulz, 1984). Due to the fact that most of the copper positions are far away from full occupancy we will only give qualitative data for the opps.

In good agreement with the copper distribution derived from jpdf analysis, the highest opps of all argyrodites were observed for the group 15 members of the Type 1 argyrodites. Values between $0.6 \mathrm{eV}$ and $1.3 \mathrm{eV}$ for pathway 1 and pathway 2 are well above $0.3 \mathrm{eV}$. The latter activation energy is the maximum value that should be observed for good or "super" ionic conductors according to Agrawal and Gupta (1999). A higher copper content per formula unit for the group 14 members of the Type 1 argyrodites results in significant smaller opps (0.1$0.2 \mathrm{eV}$ ), well below the value of $0.3 \mathrm{eV}$. The maximum copper distance $d_{\max }(\mathrm{Cu}-\mathrm{Cu})$ along a pathway is almost the same for the two pathways. For instance the distances vary between $2.36 \AA$ and $2.53 \AA$ (group 15 members) and between $2.41 \AA$ and $2.55 \AA$ (group 14 members) for pathway 1 . The same narrow distance distribution is observed for pathway 2. Obviously the copper content does not have a significant influence on $d_{\max }(\mathrm{Cu}-\mathrm{Cu})$ and therefore the maximum jump distance can not be a decisive factor for the change of the opp. Other factors influencing the occupancy of the copper positions and the coordination of copper along the diffusion pathway obviously play an important role and have to be taken into account.

The Type 2 and Type 4 argyrodites show comparable opps than the Type 1 (group 14) argyrodites. Slightly smaller opps $(<0.1 \mathrm{eV})$ were observed for the Type 3 argyrodites. According to the results from opp analyses a copper diffusion seems to be possible for all copper argyrodites and the argyrodites can be ranked in terms of increasing copper mobility:

Type 1 (group 15) < Type 1 (group 14)

$\cong$ Type $2 \cong$ Type $4<$ Type 3

Type 3 argyrodites therefore seem to be the most promising candidates for high ionic conductivity.
Table 7. Total conductivities of quaternary copper argyrodites.

\begin{tabular}{llll}
\hline $\begin{array}{l}\text { Compound Conductivity } \\
\left(\Omega^{-1} \mathrm{~cm}^{-1}\right)\end{array}$ & Type & Literature \\
\hline $\mathrm{Cu}_{6} \mathrm{PS}_{5} \mathrm{Br}$ & $1 \cdot 10^{-5} / 1.2 \cdot 10^{-5}$ & 1 (group 15) & $\begin{array}{l}\text { Kuhs et al. (1979)/ } \\
\text { Studenyak et al. 1997 }\end{array}$ \\
$\mathrm{Cu}_{6} \mathrm{PS}_{5} \mathrm{I}$ & $5 \cdot 10^{-5} / 1.3 \cdot 10^{-3}$ & 1 (group 15) & $\begin{array}{l}\text { Kuhs et al. (1979)/ } \\
\text { Studenyak et al. (1997) }\end{array}$ \\
$\mathrm{Cu}_{6} \mathrm{PS}_{5} \mathrm{Cl}$ & $8 \cdot 10^{-4} / 4.3 \cdot 10^{-2}$ & 2 & $\begin{array}{l}\text { Kuhs et al. (1979)/ } \\
\text { Studenyak et al. (1997) }\end{array}$ \\
$\mathrm{Cu}_{7} \mathrm{GeS}_{5} \mathrm{I}$ & $5.6 \cdot 10^{-3}$ & 1 (group 14) & \begin{tabular}{l} 
Studenyak et al. (2002) \\
\hline
\end{tabular} \\
\hline
\end{tabular}

Ionic conductivity was observed at least for some of the argyrodites under discussion, e.g. for $\mathrm{Cu}_{6} \mathrm{PS}_{5} X$ $(X=\mathrm{Cl}, \mathrm{Br}, \mathrm{I})$ and $\mathrm{Cu}_{7} \mathrm{GeS}_{5} \mathrm{I}$ (Kuhs et al., 1979; Studenyak et al., 1997 and 2002). The predicted trend of equation (1) can be directly correlated with the measured conductivity reported by Kuhs et al. (1979), and Studenyak et al. (1997, and 2002). Estimated conductivities at $300 \mathrm{~K}$ are summarized in Table 7.

\section{Conclusion}

The crystal structures of the high temperature modifications of 12 copper argyrodites were discussed with the focus on the distribution of the mobile copper ions. The argyrodites are classified into four different types according to the differences concerning the copper coordination. A new and illustrative structure description using centred, face sharing Friauf polyhedra which are separated into sets of double tetrahedra was developed to discuss the complex copper distribution of the argyrodites. Physical properties and unexpected trends of the ionic conductivities can be understood in terms of this new classification. A combined analysis of joint probability density functions and one particle potentials results in a prediction of potential ionic conductors.

Acknowledgments. This work was financially supported by the Deutsche Forschungsgemeinschaft (DFG).

\section{References}

Agrawal, R. C.; Gupta, R. K.: Superionic solids: composite electrolyte phase - an overview. J. Mat. Sci. 34 (1999) 1131-1162.

Bachmann, R.; Schulz, H.: Anharmonic Potentials and Pseudo Potentials in Ordered and Disordered Crystals. Acta Crystallogr. A40 (1984) 668-675.

Batirov, T. M.; Fridkin, V. M.; Nitsche, R.; Verkhovskaya, K. A.: Bulk photovoltaic and illumination effects in the argyrodite-type ionic conductors copper phosphide sulfide bromide $\left(\mathrm{Cu}_{6} \mathrm{PS}_{5} \mathrm{Br}\right)$ and copper phosphide sulfide iodide $\left(\mathrm{Cu}_{6} \mathrm{PS}_{5} \mathrm{I}\right)$. Phys. Status Solidi A: Applied Research 72 (1) (1982) K105-K108.

Belin, R.; Aldon, L.; Zerouale, A.; Belin, C.; Ribes, M.: Crystal structure of the non-stoichiometric argyrodite compound $\mathrm{Ag}_{7-x} \mathrm{GeSe}_{5} \mathrm{I}_{1-x}(x=0.31)$. A highly disordered silver superionic conducting material. Solid State Sci. 3 (2001) 251-265.

DIAMOND - Visual Crystal Structure Information System, Version 2.1e, Crystal Impact Postfach 1251, D-53002 Bonn, Germany (2001).

Gaudin, E.; Deiseroth, H. J.; Zaiss, T.: The argyrodite gamma$\mathrm{Ag}_{9} \mathrm{AlSe}_{6}$ : A non-metallic filled Laves phase. Z. Kristallogr. 216 (1) (2001) 39-44.

Goldschmidt, V. M.: Argyrodite from Bolivia. Z. Kristallogr. Mineral. 45 (1909) 548-554. 
Gulay, L. D.; Olekseyuk, I. D.; Parasyuk, O. V.: Crystal structure of the $\mathrm{Hg}_{4} \mathrm{SiS}_{6}$ and $\mathrm{Hg}_{4} \mathrm{SiSe}_{6}$ compounds. J. Alloys Compd. 347 (2002) $115-120$.

Hahn, H.; Schulze, H.; Sechser, L.: Über einige ternäre Chalkogenide vom Argyrodit-Typ. Naturwissenschaften 52 (1965) 451.

Krebs, B.; Mandt, J.: Structure and properties of cadmium thiosilicate $\left(\mathrm{Cd}_{4} \mathrm{SiS}_{6}\right)$ and cadmium selenosilicate $\left(\mathrm{Cd}_{4} \mathrm{SiSe}_{6}\right)$. Z. Anorg. Allg. Chem. 388 (3) (1972) 193-206.

Kuhs, W. F.; Nitsche, R.; Scheunemann, K.: The crystal structure of $\mathrm{Cu}_{6} \mathrm{PS}_{5} \mathrm{Br}$, a new superionic conductor. Acta Crystallogr. B34 (1978a) 64-70.

Kuhs, W. F.: Die Verbindungen $\mathrm{Cu}_{6} \mathrm{PS}_{5} \mathrm{Hal}(\mathrm{Hal}=\mathrm{Cl}, \mathrm{Br}$, I) und $\mathrm{Cu}_{7} \mathrm{PS}_{6}$. Mitglieder einer neuen Strukturfamilie ionenleitender (Halogenid-)Chalkogenide mit ikosaedrisch gepackten Anionen. Phd Thesis, University of Freiburg (1978b).

Kuhs, W. F.; Nitsche, R.; Scheunemann, K.: The Argyrodites - A new family of tetrahedrally closed packed structures. Mater. Res. Bull. 14 (1979) 241-248.

Kuhs, W. F.: Generalised atomic displacements in crystallographic structure analysis. Acta Crystallogr. A48 (1992) 80-98.

Matje, P.; Schön, G.: Silver tin telluride $\left(\mathrm{Ag}_{8} \mathrm{SnTe}_{6}\right)$ - a new substitute of argyrodite. Z. Naturforsch. B35 (2) (1980) 247-249.

Nilges, T.; Reiser, S.; Hong, J. H.; Gaudin, E.; Pfitzner, A.: Preparation, structural, Raman and impedance spectroscopic characterisation of the silver ion conductor $(\mathrm{AgI})_{2} \mathrm{Ag}_{3} \mathrm{SbS}_{3}$. Phys. Chem. Chem. Phys. 4 (2002) 5888-5894.

Ohba, T.; Kitano, Y.; Komura, Y.: The Charge-Density Study of the Laves Phases, $\mathrm{MgZn}_{2}$ and $\mathrm{MgCu}_{2}$. Acta Crystallogr. C40 (1984) $1-5$.

Onoda, M.; Wada, H.; Hiroaki, T.; Tansho, M.; Ishii, M.: Low-temperature phases (phase II) of ionic conductors $\mathrm{Ag}_{7} \mathrm{TaS}_{6}$ and $\mathrm{Ag}_{7} \mathrm{NbS}_{6}$. Solid State Ionics 113-115 (1998) 515-519.

Palache, C.; Berman, H.; Fondel, C.: Dana's system of Mineralogy. $7^{\text {th }}$ edition (1944) $356 \mathrm{ff}$.
Pepke, E.; Murray, J.; Lyons, J.; Hwu, T.-Y: SCIAN, Scientific Visualisation Package, Version 1.1a, Florida State University, USA (1994).

Petříček, V.; Dusek, M.: The crystallographic computing system JANA98. Institute of Physics, Praha, Czech Republic (1998).

Pfitzner, A.: $(\mathrm{CuI})_{2} \mathrm{Cu}_{3} \mathrm{SbS}_{3}$ : Copper iodide as solid solvent for thiometalate ions. Chem. Eur. J. 3 (12) (1997) 2032-2038.

Studenyak, I. P.; Stefanovich, V. O.; Kranjcec, M.;.Desnica, D. I.; Azhniuk, Yu. M.; Kovacs, Gy. Sh.; Panko, V. V.: Raman scattering studies of $\mathrm{Cu}_{6} \mathrm{PS}_{5} \mathrm{Hal}(\mathrm{Hal}=\mathrm{Cl}, \mathrm{Br}$ and $\mathrm{I})$ fast-ion conductors. Solid State Ionics 95 (1997) 221-225.

Studenyak, I. P.; Kranjcec, M.; Kovacs, Gy. Sh.; Desnica-Frankovic, I. D.; Molnar, A. A.; Panko, V. V.; Slivka, V. Yu.: Electrical and optical absorption studies of $\mathrm{Cu}_{7} \mathrm{GeS}_{5} \mathrm{I}$ fast-ion conductor. J. Phys. Chem. Sol. 63 (2002) 267-271.

Susa, K.; Steinfink, H.: $\mathrm{GeCd}_{4} \mathrm{~S}_{6}$, a new Tetrahedral Structure Type. Inorg. Chem. 10 (1971) 1754-1756.

Tansho, M.; Wada, H.; Ishii, M.; Onoda, Y.: Silver ionic conductor $\mathrm{Ag}_{9} \mathrm{GaSe}_{6}$ studied by $\mathrm{Ag}$ and Ga NMR. Solid State Ionics 86-8 (1996) 155-158.

Wada, H.: Crystal structures and silver ionic conductivities of the new compounds silver niobium sulfide $\left(\mathrm{Ag}_{7} \mathrm{NbS}_{6}\right)$, silver tantalum selenide $\left(\mathrm{Ag}_{7} \mathrm{TaSe}_{6}\right)$ and silver tantalum selenide iodide $\left(\mathrm{Ag}_{7-x} \mathrm{TaSe}_{6-x} \mathrm{I}_{x}\right)(0.1<x<0.5)$. J. Alloys Compd. 178 (1992) $315-323$.

Wada, H.; Sato, A.; Onoda, M.; Adams, S.; Tansho, M.; Ishii, M.: Phase transition and crystal structure of silver-ion conductor $\operatorname{Ag}_{12-n} M^{+n} \mathrm{~S}_{6}(M=\mathrm{Ti}, \mathrm{Nb}, \mathrm{Ta})$. Solid State Ionics 154-155 (2002) 723-727.

XRED and XSHAPE, Programmes for numerical absorption correction. Stoe and Cie GmbH, Darmstadt, Germany (1999).

Zucker, U. H.; Schulz, H.: Statistical approaches for the treatment of anharmonic motion in crystals. A comparison of the most frequently used formalisms of anharmonic thermal vibrations. Acta Crystallogr. A38 (1982) 563-568. 